\title{
TOPICS IN STATISTICAL MECHANICS
}

\author{
Veit Elser \\ Ph. D. Thesis \\ Lawrence Berkeley La'ooratory \\ and \\ Department of Physics \\ University of California \\ Berkeley, California 94720
}

This work was supported by the Director, Office of Energy Research, Office of High Energy and Nuclear Physics, Division of High Physics of the U.S. Department of Energy under Contract DE-AC03-76SF00098. 


\title{
TOPICS IN STATISTICAL MECHANICS
}

\author{
Veit Elser
}

\begin{abstract}
This thesis deals with four independent topics in statistical Imechanics:

(1) The dimer problem is solved exactly for a hexagonal lattice with general boundary using a known generating function from the thec $\gamma$ of partitions. It is shown that the leading term in the entropy depenc on the shape of the boundary.

(2) Continuum models of percolation and self-avoiding walks are introduced with the property that their series expansions are sums over linear graphs with intrinsic combinatorial weights and explicit dimension dependence.

(3) A constrained SOS model is used to describe the edge of a simple cubic crystal. Low and high temperature results are derived as well as the detailed behavior near the crystal facet.

(4) The microscopic model of the $\lambda$-transition involving atomic permutation cycles is reexamined. In particular, a new derivation of the twocomponent fleld theory model of the critical behavior is presented. Results for a lattice model originally proposed by Kikuchi are extended with a high temperature series expansion and Monte Carlo simulation.
\end{abstract}




\section{ACKNOWLEDGEMENTS}

I would like to thank the various people who were my designated advisors at various times for allowing me to pursue an independent style of research. I am particularily grateful to Orlando Alvarez and Robert Cahn for their unsolicited interest, advice, and encouragement.

A considerable fraction of this work would not have been possible without the excellent resources of the Lawrence Berkeley Laboratory. I especially thank the Special Function Division and its able staff, particularily Ghassan Batrouni, Joe Carlson, Steve Sharpe, Adlai Smith, and Matt Visser for bailing me out of numerous technical difficulties including boredom.

Finally, I thank my parents for encouraging me to excel in fingerpainting rather than penmanship and Liz Hill for having had the courage to roller-skate.

This work was supported by the Director, Office of Energy Research, Office of High Energy and Nuclear Physics, Division of High Energy Physics of the U. S. Department of Energy under Contract DE-AC0376SF00098. 


\section{TABLE OF CONTENTS}

ACKNOWLEDGEMENTS i

TARLE OF CONTENTS ii

DINERS ON A HEXAGONAL LATTICE WTTH BOUNDARY 1

References 7

$\begin{array}{ll}\text { Figures } & 8\end{array}$

GAUSSIAN-CLUSTER MODELS OF PERCOLATION AND SELF-AVODING WALKS 11

$\begin{array}{ll}\text { Introduction } & 12\end{array}$

$\begin{array}{ll}\text { Percolation } & 13\end{array}$

Self-Aroiding Walks $\quad 20$

Appendix $\quad 23$

$\begin{array}{ll}\text { References } & 26\end{array}$

$\begin{array}{ll}\text { Table } & 27\end{array}$ 
THE EQUILIBRIUM SHAPE OF CRYSTAL EDGES 28

$\begin{array}{ll}\text { 1. Introduction } & 29\end{array}$

2. Low Temperature Expansion 32

3. High Temperature Limit 37

4. Low Density Expansion 41

References $\quad 48$

$\begin{array}{ll}\text { Figures } & 49\end{array}$

THE LOOP GAS PICTURE OF THE $\lambda$-TRANSITION 50

1. Introduction 51

2. Ideal Bose Condensation in Configuration Space 52

3. Off Diagonal Long Range Order 59

4. The Dilute Hard Sphere Gas 61

5. The Origin of $n=2 \quad 70$

6. A Lattice Model of the Loop Gas 71

$\begin{array}{ll}\text { References } & \mathbf{7 9}\end{array}$

$\begin{array}{ll}\text { Figures } & 80\end{array}$ 
DIMERS ON A HEXAGONAL LATTICE WITH BOUNDARY 
Implicit in the notion of a thermodynamic limit is the idea that bulk properties are insensitive to the precise nature of the boundary conditions imposed. An instructive example is provided by the dimer problem on a square lattice. When the dimers are required to occupy every site of the lattice the prohiem can be solved exactly both for the case of an $m \times n$ rectangular boundary ${ }^{1,2}$ as well as for the $m \times n$ torus $^{2}$. With either choice of boundary conditions one finds a bulk entropy per dimer:

$$
\frac{1}{\frac{1}{2} m n} \log Z \sim \frac{2}{\pi}\left(1-\frac{1}{3^{2}}+\frac{1}{5^{2}}-\frac{1}{7^{2}}+\cdots\right) \quad m, n \rightarrow \infty
$$

In this note it will be shown that the above statement does not hold for the dimer problem on the hexagonal lattice. An exact solution has been known for some time ${ }^{3}$ but only for the case of toroidal boundary conditions where one does obtain a bulk entropy ( $N=$ number of dimers):

$$
\begin{aligned}
\frac{1}{N} \log Z & \sim \frac{2}{\pi} \int_{0}^{\pi / 3} \log (2 \cos x) d x \quad N \rightarrow \infty \\
& =.338314
\end{aligned}
$$

If instead the problem is formuiated in a general hexagonal region (to be described below) it is seen to be equivalent to the combinatorial problem of "plane partitions" 4 . Fortunately, the generating function for plane partitions is known. When the result is applied to the dimer problem it will be seen that a bulk limit of the entropy does not exist.

Figure 1 shows a typical dimer configuration. The dimers are placed along the edges of the lattice and are refresented by double bonds. Since the maximum dimer density corresponds to each site being the endpoint of exactly one double bond, the resulting configurations are equivalent to the possible Kekule structures of carbon-carbon bonds in graphite. 
Consider now a description in t.erms of the dual triangular lattice. The hexagonal lattice sites are mapped into the centers of triangles; the double bonds now joining adjacent triangles in the triangular lattice. Since every triangle is joined to exactly one adjacent triangle, the dimer configuration is equivalent to a tiling of the triangular lattice with "triangular dominoes", or rhombi (see Fig. 2).

The tiling description will now be mapped into a three-dimensional structure. The three orientations of rhombi can be generated by projecting faces of a cube into a plane perpendicular to the cube's main diagonal. Figure 3 depicts a collection of unit cubes arranged in one octant of a threedimensional coordinate system (portions of the planes $x=0, y=0$ and $z=0$ are shown as well). By projecting only the "non-hidden" surfaces of Fig. 3 into the (111) plane, we produce the tiling pattern of Fig. 2. The arrangement of unit cubes at integer points of the coordinate system follows uniquely from the tiling pattern. We proceed by building up layers of cubes. The dashed line from $A$ to $A^{\prime}$ in Fig. 2 instructs us how to build up the layer for $0<y<1$. It consists of stacks of 3,2 and 1 cubes in the $z$-direction as we proceed in the positive $x$ direction. The dashed line $A A^{\prime}$ is found by connecting opposite sides of the rhombi. Similarily, the layer $1<y<2$ is generated by following line $B B^{\prime}$, etc. It is easy to see that the stacks of cubes always have nonincreasing height as we proceed in the posivive $x$-direction. If instead we had analyzed the configuration in terms of layers of constant $x$ (by proceeding from $C$ to $C^{\prime}$, etc.) we would have found that the heights of the stacks of cube; are also non-increasing in the positive $y$-direction. Figure 4 shows the heights of the stacks of cubes in the $x-y$ plane. We have just shown that dimer configurations of the original hexagonal lattice correspond uniquely to the assignment of integers $0,1,2$ or 3 to a $3 \times 4$ table such that both rows and columns form non-increasing sequesces. 
The general problem described above, of counting the number of sets of $k \times /$ integers $\left\{z_{i j}\right\}$ satisfying

$$
\begin{aligned}
& 0 \leq z_{i j} \leq m \\
& z_{i j} \leq z_{i+1 j} \\
& z_{i j} \leq z_{i j+1}
\end{aligned}
$$

is the problem of plane partitions treated extensively by MacMahon". From the three-dimensional representation of Fig. 3 it is clear that the counting of configurations is completely symmetric with respect to permuting $k, l$ and $m$. In our case, $k, l$ and $m$ measure the sides of the hexagon shown in Fig. 2. Moreover, the number of dimers belonging to the three differen $\imath$ orientations are simply $k \cdot l, l \cdot m$ and $m \cdot k$. MacMahon discovered the generating function for plane partitions:

$$
\begin{gathered}
G_{k l m}(x)=\sum_{M=0}^{k l m} P_{M} x^{M} \\
=\frac{F_{k+l+m}(x) F_{k}(x) F_{l}(x) F_{m}(x)}{F_{k+l}(x) F_{1+m}(x) F_{m+k}(x)} \\
F_{n}(x)=(1-x)^{n-1}\left(1-x^{2}\right)^{n-2} \cdots\left(1-x^{n-1}\right)
\end{gathered}
$$

Here $P_{M}$ is the number of configurations subject to the constraint

$$
\sum_{\substack{1 \leq i \leq k \\ 1 \leq j \leq 1}} z_{i j}=M
$$


For example,

$$
\begin{gathered}
G_{222}(x)=1+x+3 x^{2}+3 x^{3}+4 x^{4}+3 x^{5}+3 x^{6}+x^{7}+x^{8} \\
G_{222}(1)=20 .
\end{gathered}
$$

We will evaluate $G_{k l m}$ for $x=1$ when $k, l$ and $m$ are all large. Setting $x=e^{-t}$ we have,

$$
\log F_{n}=\sum_{p=1}^{n-1}(n-p) \log \left(1-e^{-p i}\right)
$$

In the limit $t \rightarrow 0$ for $n$ large but fixed,

$$
\begin{aligned}
\log F_{n} & \sim \frac{1}{t^{2}} \int_{0}^{n t}(n t-u) \log \left(1-e^{-u}\right) d u \\
& \sim \frac{1}{2} n^{2} \log n t .
\end{aligned}
$$

Substituting into the expression for $G_{k l m}$ we find:

$$
\begin{gathered}
S_{k l m}=\log G_{k l m}(1) \sim N s(x, y, z) \\
N=k l+l m+m k \\
s(x, y, z)=\frac{1}{2(x y+y z+z x)}\left[x^{2} \log x+y^{2} \log y+z^{2} \log z\right. \\
\left.-(1-x)^{2} \log (1-x)-(1-y)^{2} \log (1-y)-(1-z)^{2} \log (1-z)\right] \\
n=k+l+m, \quad x=\frac{k}{n}, \quad y=\frac{l}{n}, \quad z=\frac{m}{n}
\end{gathered}
$$

We observe that first of all the boundary completely determines the orientational distribution of the dimers. Moreover, the speciflc entropy 
given by the function $s(x, y, z)$ is not a constant but clearly depends on the shape of the boundary. The maximum entropy per dimer is obtained when the bouzdary is a regular hexagon:

$$
s\left(\frac{1}{3}, \frac{1}{3}, \frac{1}{3}\right)=\frac{3}{2} \log 3-2 \log 2=.2616241
$$

Since the dimer conflgurations considered here are a proper subset of those counted in the toroidal problem, the entropy (2) could not have been greater than (1).

In order to understand the difference between the square and hexagonal dimer problems when boundaries are present we focus on the nature of conflgurations near a single straight boundary. In Fig. 5, line $\ell_{0}$ describes the boundary and has no dimers crossing over it. Line $\ell_{1}$ may be crossed by at most one dimer, line $\ell_{2}$ by at most two, and so on. Now if at some distance away from the boundary, say near the line $\ell_{k}$, the dimer configurations were representative of a bulk sample, then a fixed fraction of $i_{k}$ would be crossed by dimers. By the previous remark this requires that $k$ be proportional to the length of the boundary. Thus the region between $l_{0}$ and $l_{k}$ where the dimers are not representative of bulk properties does not become negligible when we take the thermodynamic limit. We see in Fig. 6 that the square lattice has a very different behavior. Already at line $\ell_{1}$ the number of crossing dimers can be close to the bulk value. 


\section{Acknowledgments}

The author would like to thank Liz Hill for help with the illustrations. This work was supported by the Director, Offce of Energy Research, Office of High Energy and Nuclear Physics, Division of High Energy Physics of the U. S. Department of Energy under Contract DEAC03-76SF00098.

\section{References}

(1) M. E. Fisher Phys. Rev. $124 ! 664$ (1961)

(2) P. W. Kasteleyn Physica 27 I209 (1961)

(3) G. H. Wannier Phys. Rev. 79357 (1950)

P. W. Kasteleyn J. Math. Phys. 4287 (1963)

F. Y. Wu Phys. Rev. 168539 (1968)

J. F. Nagle Phys. Rev. Letters 341150 (1975)

(4) P. A. MacMahon "Combinatory Analysis" Vol. 2, Cambridge University Press 1916

T. W. Chaundy Quart. J. Math. 2234 (193i) 


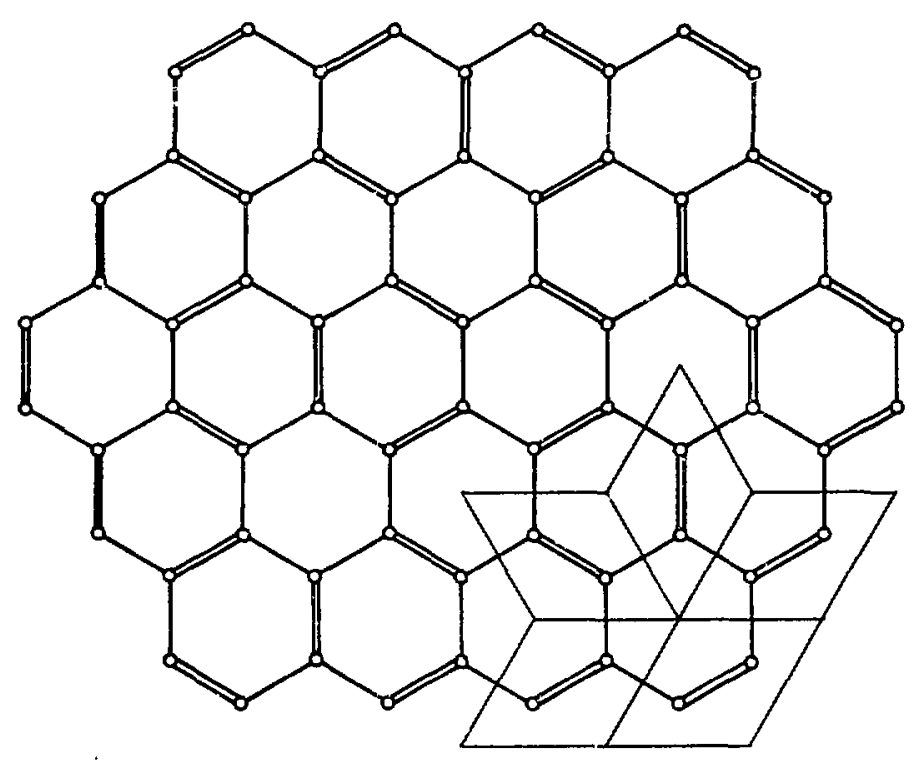

Figure 1

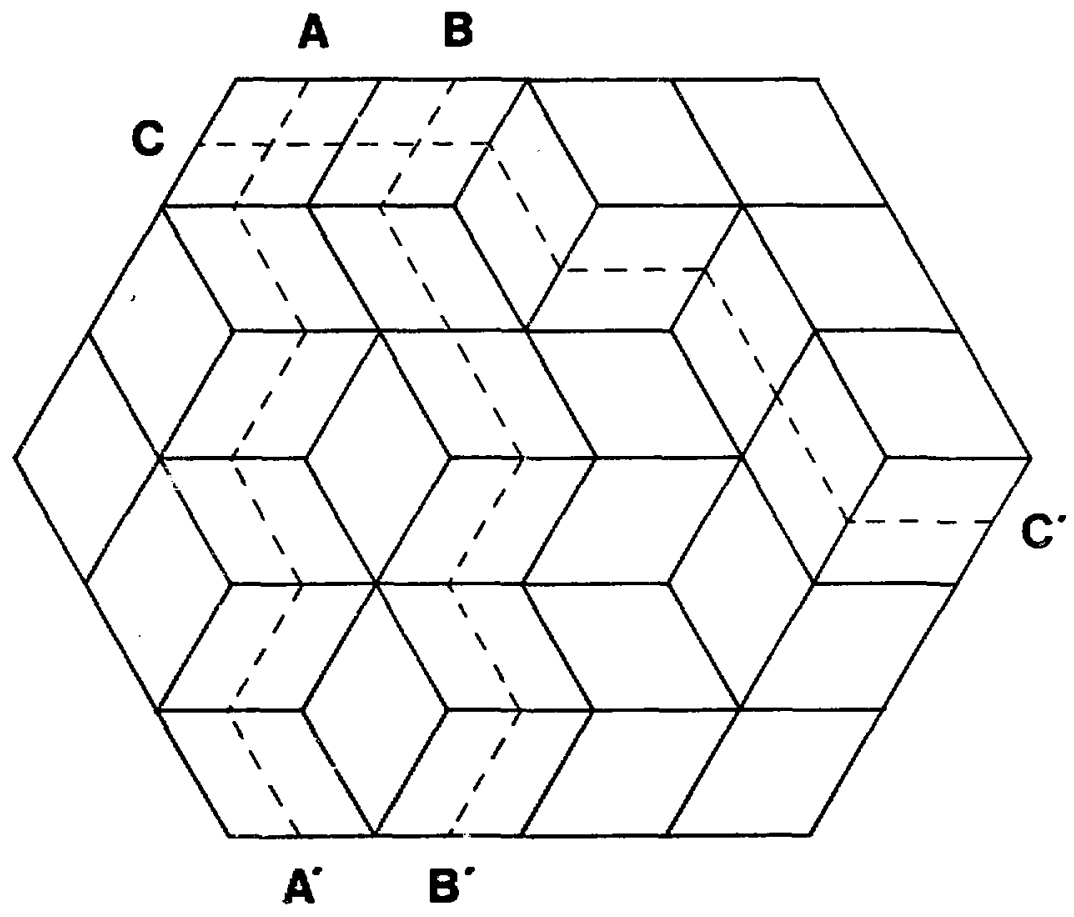

Figure 2 


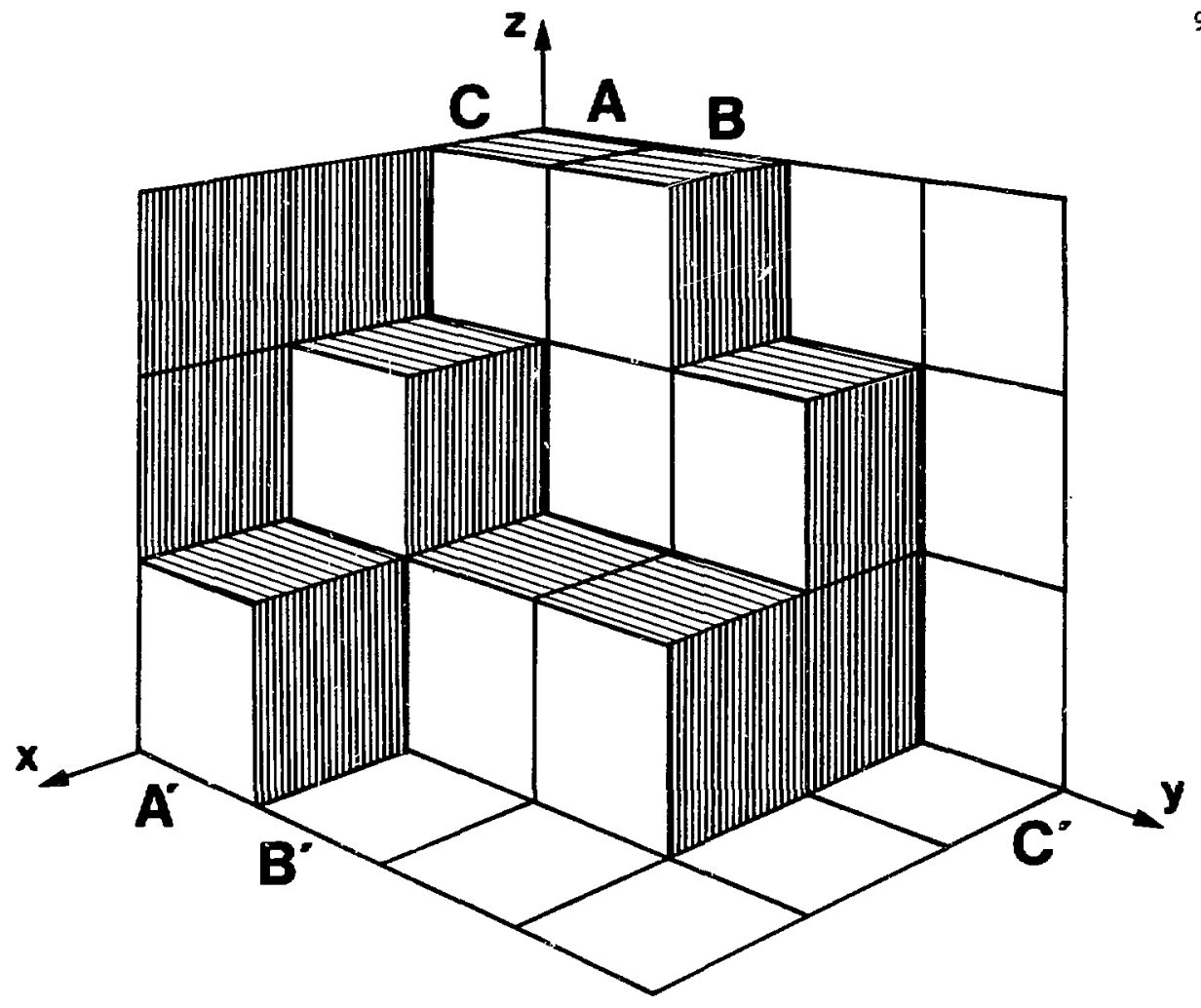

Figure 3

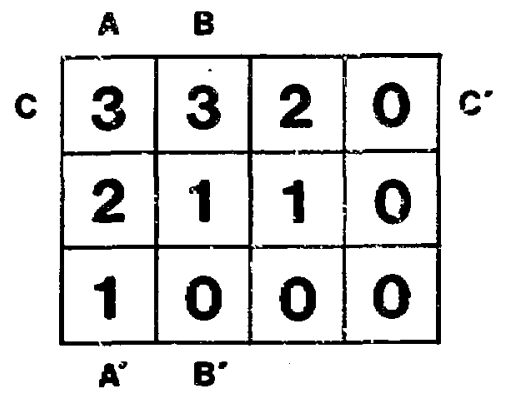

Figure 4 


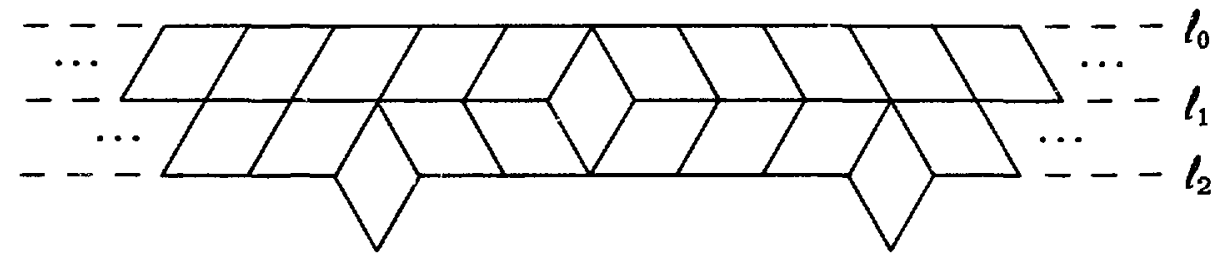

Figure 5

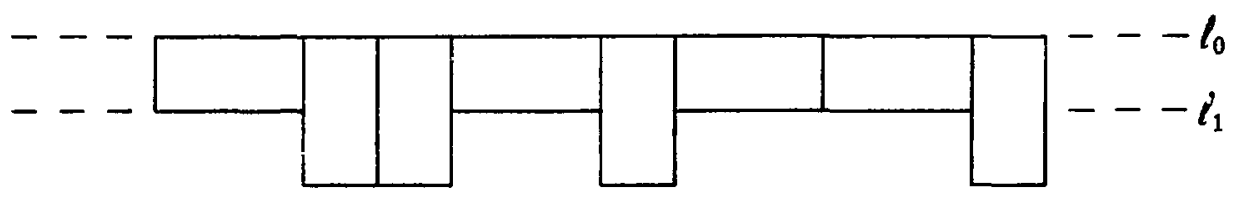

Figure 6 
GAUSSIAN-CLUSTER MODELS OF PERCOLATION AND SELF-AVOIDING WALKS 


\section{Introduction}

The importance of dimensionality in statistical mechanics has motivated the study of a number of systems where the dimensionality $D$ is a freely adjustable parameter. Two examples are the $D$-dependence of momentum (loop) integrals in field-theoretic models ${ }^{1}$ and the series expansions for models defined on a $D$-dimensional hypercubic lattice where $D$ also appears explicitly ${ }^{2}$. This paper introduces models of percolation and self-avoiding walks where series expansions exhibit a $D$-dependence in yet another way.

The basic idea is to exploit a well known property of gaussian integrals $^{3}$. Let $x_{1}, \ldots, x_{n}$ be $D$-dimensional cartesian coordinates of $n$ points and let $C$ be a simple connected graph having $n$ vertices labeled $1,2, \ldots, n$. If we associate each edge of $C$ with the pair of vertices it connects, then

$$
\int d^{D} x_{2} \int d^{D} x_{3} \cdots \int d^{D} x_{n} \prod_{\text {edges }} \exp \left(-\left(x_{i}-x_{j}\right)^{2}\right)=\left(\frac{\pi}{\tau(C)}\right)^{D / 2},
$$

where $\tau(C)$ denotes the number of spanning trees of $C$. Thus, if a problem can be formulated in the contiruum in such a way that the cluster integrals appearing in scries expansions are always of the above type, the task is reduced to combinatorics. This strategy has been used previously by Ford and Uhlenbeck ${ }^{4}$ in their study of a gas of particles having the pairwise interaction $-\beta V(r)=\log \left(1-\exp \left(-a r^{2}\right)\right)$. 


\section{Percolation}

Consider the percolation problem defined on a set of $N$ points $x_{1}, \ldots, x_{N}$ distributed uniformly at random inside a $D$-dimensional volume $V$. Clusters are delined by establishing connections (adjacency) between certain pairs of points. Specifically, points $x_{i}$ and $x_{j}$ are connected by an "cdge" with probability $\exp \left(-a\left(x_{i}-x_{j}\right)^{2}\right)$. Here $a$ plays the role of the bond probability and controls the size of clusters when one maintains a fixed density $\rho=N / V$. Equivalently, fixing $a$ and varying $\rho$ reproduces the site-type problem.

Our approach to analyzing this model will be to obtain the socalled "cluster numbers" ${ }^{5}$. A $k$-cluster is defined to be an edge-connected set of $k$ points having no edge connections with any of the other $N-k$ points. Dividing the average number of $k$-clusters present in the volume by $N$ gives the cluster numbers $\bar{n}_{k}\left(x_{1}, \ldots, x_{N}\right)$. Averaging this quantity over all ensembles of points gives the cluster numbers appropriate to a uniform, random distribution of points:

$$
n_{k}=\frac{1}{V^{N}} \int d^{D} x_{1} \ldots \int d^{D} x_{N} \bar{n}_{k}\left(x_{1}, \ldots, x_{N}\right)
$$

We will obtain $n_{k}$ by considering $p_{k}$, the probability that a given point, call it $y_{1}$, belongs to a $k$-cluster (ag in, averaged over ensembles). The cluster numbers then follow from $n_{k}=p_{k} / k$. There are

$$
\left(\begin{array}{c}
N-1 \\
k-1
\end{array}\right)
$$

ways of choosing the other $k-1$ points of the cluster, call them $y_{2}, \ldots, y_{k}$. Once chosen, the set of possible $k$-clusters that can be formed fall into a one-one correspondence with the set of simple connected graphs on $k$ (labelcd) vertices, $\mathcal{C}_{k}$. For a particular graph $C \in \mathcal{C}_{k}$ with vertices labeled $1, \ldots, k$, one associates the following probability factor with each pair of 
vertices $1 \leq i<j \leq k$ :

$$
P_{i j}(C)= \begin{cases}\exp \left(-a\left(y_{i}-y_{j}\right)^{2}\right) & \text { if } i \text { and } j \text { are adjacent; } \\ 1-\exp \left(-a\left(y_{i}-y_{j}\right)^{2}\right) & \text { otherwise. }\end{cases}
$$

The probability of the cluster conflguration is given by the product of these factors multiplied by

$$
\prod_{i=1}^{N-k} \prod_{j=1}^{k}\left[1-\exp \left(-a\left(z_{i}-y_{j}\right)^{2}\right)\right]=\prod_{i=1}^{N-k}\left(1+f\left(z_{i}\right)\right),
$$

where $z_{1}, \ldots, z_{N-k}$ are the points not included in the cluster. In the limit $N \rightarrow \infty$ with $\rho$ and $k$ fixed, the integrations over the positions $z_{1}, z_{2}, \ldots$ can be evaluated in closed form. However, in following the strategy of utilizing expressions involving only gaussian integrals, we will be interested in expanding in powers of the density:

$$
\begin{gathered}
\frac{1}{V^{N-k}} \int d^{D} z_{1} \cdots \int d^{D} z_{N-k} \prod_{i=1}^{N-k}\left(1+f\left(z_{i}\right)\right) \rightarrow \exp \left(\rho \int d^{D} z f(z)\right) \\
=1+\rho \int d^{D} z_{1} f\left(z_{1}\right)+\frac{\rho^{2}}{2 !} \int d^{D} z_{1} \int d^{D} z_{2} f\left(z_{1}\right) f\left(z_{2}\right)+\cdots
\end{gathered}
$$

When (4) is multiplied by the factors (3), the binomial coefficient (2) (with $N \rightarrow \infty, k$ fixed) and averaged over the positions $y_{1}, \ldots, y_{k}$, one obtains the probability $p_{k}(C)$ that $y_{1}$ belongs to a $k$-cluster with topology specified by $C$. Summing $p_{k}(C)$ over all possible connected graphs $C \in \mathcal{C}_{k}$ and taking into account the relationship between $n_{k}$ and $p_{k}$ we arrive at the expansion

$$
\begin{array}{r}
n_{k}=\frac{\rho^{k-1}}{k !} \sum_{C \in \mathcal{C}_{k}}\left\{\sum_{n=0}^{\infty} \frac{?^{n}}{n !} \int d^{D} y_{2} \cdots \int d^{D} y_{k} \int d^{D} z_{1} \cdots \int d^{D} z_{n}\right. \\
\left.\prod_{1 \leq i<j \leq k} P_{i j}(C) \prod_{i=1}^{n} f\left(z_{i}\right)\right\}
\end{array}
$$


where,

$$
f(z)=\prod_{i=1}^{k}\left[1-\exp \left(-a\left(z-y_{i}\right)^{2}\right)\right]-1
$$

We see that (5) is a sum of terms of the form (1); the coefficient cf

$$
\left[\rho\left(\frac{\pi}{a}\right)^{D / 2}\right]^{k+n-1}=x^{k+n-1}
$$

involving contributions from connected graphs having $k+n$ vertices. With this in mind, we will consider an expansion of the generating function

$$
\mathrm{F}(x, y)=\sum_{k=1}^{\infty} y^{k} n_{k}(x)
$$

organized in the form

$$
F(x, y)=\sum_{m=1}^{\infty} \frac{x^{m-1}}{m !}\left\{\sum_{C \in C_{m}}\left(\frac{1}{r(C)}\right)^{D / 2} W(C ; y)\right\}
$$

where $W(C ; y)$ is a polynomial of degree bounded by the number of vertices of $C$.

We now turn to the problem of evaluating $W(C ; y)$ for some graph $C \in C_{k+n}$. In particular, consider the contributions to the coefficient of $y^{k}$. These are due to various graphs $N \in \mathcal{C}_{k}$ that characterize $k$-cluster topologies as well as the different ways these graphs might be embedded in $C$. To be precise, let $N$ be a subgraph of $C$ (written $N \subseteq C$ ). Denote by $C-N$ the subset of $C$ where all the vertices of $N$ as well as the edges incident to thein have been deleted. If $C-N$ is not empty then $C-N$ is a subgrapl of $C$. A subgraph $N \subseteq C$ that corresponds to an acceptable cluster embedding will be called a nucleus and has the properties: 
(i) $N$ is connected;

(ii) $C-N$ has no edges. (Equivalently: the vertices of $N$ constitute a covering of $C$; i.e. at least one end of every edge of $C$ is a vertes of $N$.)

The vertices not in the nucleus should be identified with the points $z_{1}, \ldots, z_{n}$ outside the cluster, which by (5) are not mutually interconnected by ganssian factors. In (5), the nucleus belongs to a distinguished subset of the $k+n$ vertices of $C$. If we relax this condition on the embedding we will be overcounting by the factor $\left(\begin{array}{c}k+n \\ n\end{array}\right)$. To ccmpensate for this we divide by the same factor in the end.

For a given cluster embedding or nucleus $N \subseteq C$, there are three types of edges $e$ of $C$. First, if $e \in N$ then $e$ contributes a gaussian factor with a plus sign according to (3a). Second, if the endpoints of $e$ belong to $N$ but $e \notin N$ then (3b) applies and the gaussian factor comes with a minus sign. Third, if one of the cndpoints of $e$ bclongs to $C-N$ then the origin of the relevant gaussian was (4) and also carries a minus sign. The parity of the contribution $\mathrm{d}$ e to a particular nucleus $N \subseteq C$ is thus

$$
(-1)^{\epsilon(C)-\epsilon(N)}
$$

where $\epsilon(G)$ denotes the number of edges of $G$.

We can now put together the total contribution of a term in (5) coming from a particular nucleus $N \subseteq C$ :

$$
\begin{aligned}
\sum_{k=1}^{\infty} y^{k} n_{k} & =\cdots+\frac{x^{k+n-1} y^{k}}{k ! n !} \frac{1}{\left(\begin{array}{c}
k+n \\
n
\end{array}\right)}(-1)^{\epsilon(C)-\epsilon(N)}\left(\frac{1}{\tau(C)}\right)^{D / 2}+\cdots \\
& =\cdots+\frac{x^{k+n-1}}{(k+n) !}\left(\frac{1}{\tau(C)}\right)^{D / 2}(-1)^{\epsilon(C)-\epsilon(N)} y^{k}+\cdots
\end{aligned}
$$

Sumıning all those terms involving a particular graph $C$ gives the weight 
polynomial

$$
W(C ; y)=\sum_{\substack{n u c l e i \\ N \subseteq C}}(-1)^{\epsilon(C)-\epsilon(N)} y^{\nu(N)},
$$

where $\nu(G)$ denotes the number of vertices of $G$.

The generating function (6) may also be expressed as a sum over nonisomorphic connected graphs,

$$
\mathrm{F}(x, y)=\sum_{\substack{\text { nonisomorphic } \\ C}} \frac{x^{\nu^{(}(C)-1}}{\sigma(C)}\left(\frac{1}{\tau(C)}\right)^{D / 2} W(C ; y),
$$

where $\sigma(G)$ is the order of the symmetry group of $G$. The first few terms of this sum, up to graphs with four vertices, are given in Table 1.

We will now prove two theorems involving the weight polynomial $W(C ; y)$. These are simple consequences of the following lemma:

I emma 1. Let $C$ be any nontrivial, simple, connected graph and let $v$ be any vertex of $C$. Then,

$$
\sum_{\{N \subseteq C \stackrel{n u c l e i}{C \mid v \in N\}}}(-1)^{\epsilon i N)}=0,
$$

where the summation is over all nuclei containing $v$.

A proof is given in the appendix.

Theorem 1. Let $C$ be a simple connected graph with at least one cut vertex $v$. Then $W(C ; 1)=0$.

Proof. Let $N \subseteq C$ be a nucleus of $C$. Suppose $v \notin N$. Then, since $N$ is a nucleus $v$ is adjacent only to vertices of $N$. Now $N$ is connected so we conclude that $C-v$ is connected contrary to the statement that $v$ is a cut vertex. Thus $v \in N$ and the statement of the theorem follows immediately from ( 7 ) and Lemma 1. 
Theorem 2. Let $C$ be a nontrivial, simple, connected fgraph. Then

$$
\frac{d}{d y} W(C ; 1)=0 .
$$

Proof.

$$
\begin{aligned}
& (-1)^{\epsilon(C)} \frac{d}{d y} W(C ; 1)=\sum_{\substack{n u c_{i l e i} \\
N \subseteq C}}(-1)^{\epsilon(N)} \nu(N) \\
& =\sum_{v \in C}\left\{\sum_{\substack{n u c l e i \\
i N \subseteq C \mid v \in N\}}}(-1)^{\epsilon(N)}\right\} \\
& =0
\end{aligned}
$$

There is another way of understanding the statement of Theorem 2. Let $P_{\text {finite }}$ be the probability that a given point in the percolation problein belongs to a finite cluster. Then

$$
\begin{aligned}
P_{\text {finite }}(x) & =\sum_{k=1}^{\infty} k n_{k}(x) \\
& =\frac{d}{d y} F(x, 1) \\
& =1
\end{aligned}
$$

with only the trivial graph contributing to the sum (6). This agrees with our expectation that $P_{\text {finite }}:=1$ on the interval $0 \leq x<x_{c}$, where $x_{c}$ is the critical density.

To obtain a nontrivial series we consider the mean cluster size, 
$\mathrm{S}(x)$, which is unity at $x=0$ and diverges at $x=x_{c}$ :

$$
\begin{aligned}
\mathrm{S}(x) & =\frac{\sum_{k=1}^{\infty} k n_{k}}{\sum_{k=1}^{\infty} n_{k}} \\
& =\left\{\sum_{\substack{\text { star } \\
S}} \frac{x^{\nu(S)-1}}{\nu(S) !}\left(\frac{1}{\tau(S)}\right)^{D / 2} W(S ; 1)\right\}^{-1}
\end{aligned}
$$

Here the weight $W(S ; 1)$ is nonzero only for graphs without cut vertices ("stars") by Theorem 1. Bclow are the lirst few terms:

$$
\begin{aligned}
S(x)^{-1}=1-\frac{1}{2} x & +\frac{1}{6}\left(\frac{1}{3}\right)^{D / 2} x^{2} \\
& +\left[\frac{1}{8}\left(\frac{1}{4}\right)^{D / 2}-\frac{1}{4}\left(\frac{1}{8}\right)^{D / 2}+\frac{1}{12}\left(\frac{1}{16}\right)^{D / 2}\right] x^{3}+\cdots
\end{aligned}
$$

The formal $D=0$ limit of $\mathrm{S}(x)$ is readily evaluated since now the gaussian integrations do not involve $\tau(S)$ but are simply \pm 1 . A convenient starting point is (5) where we firsi argue that the summation over connected graphs $C \in C_{k}$ collapses to the single term involving the complete graph $K_{k}$. Any graph with two nonadjaccnt vertices would have contained a factor (3b) yiclding two equal terms of opposite sign upon integration. Counting up the terms produced by the factors $f\left(z_{i}\right)$ we then have,

$$
\begin{gathered}
n_{k}=\frac{x^{k-1}}{k !} \sum_{n=0}^{\infty} \frac{x^{n}}{n !}\left\{\sum_{i=1}^{k}(-1)^{i}\left(\begin{array}{l}
k \\
i
\end{array}\right)\right\}^{n} . \\
=\frac{x^{k-1}}{k !} e^{-x} \quad(D=0) \\
S(x)^{-1}=\frac{1-e^{-x}}{x} .
\end{gathered}
$$


While $D=0$ is not a physically interesting case, this result does provide a useful check on the cocilicients of $S(x)^{-1}$ for general $D$.

\section{Self-Avoiding Walkg}

Let $x_{1}, \ldots, x_{n}$ be a sequence of points in $D$-dimensional space visited by a self-avoiding walk of $n-1$ steps beginning at the point $x_{1}$. As in the percolation problem, we would like to express the idea of a "chain" as well as the "excluded volume effect" in terms of gaussian factors. This can be done using the following partition function $(n \geq 2)$ :

$$
\begin{aligned}
Z_{n-1}= & \left(-\frac{1}{{ }_{\pi} D / 2}\right)^{n-1} \int d^{D} x_{2} \ldots \int d^{D} x_{n} \\
& \prod_{i=1}^{n-1} \exp \left(-\left(x_{i}-x_{i+1}\right)^{2}\right) \prod_{|i-j|>1}\left[1-\exp \left(-\left(x_{i}-x_{j}\right)^{2}\right)\right]
\end{aligned}
$$

The first product above realizes the chain constraint and by itself reproduces the behavior of the unrestricted walk if one identifies $Z_{n}$ with the number of walks of length $n-1$. The second product, over all nonconsecutive pairs of points, enforces the excluded volume constraint and renders the problem nontrivial.

Observe that every term of (9) has at most one gaussian factor connecting each pair of points. If we imagine writing out the $n$ ! copies of (9) generated by all permutations of the labels on the points and dividing by $n !$, the resulting sum in graphical language becomes:

$$
Z_{n-1}=\frac{1}{n !} \sum_{C \in C_{n}}(-1)^{\epsilon(C)-n+1} h(C)\left(\frac{1}{\tau(C)}\right)^{D / 2}
$$


Here $h(C)$ counts the number of hamiltonian paths in $C$, i.e. the number of permutations of the vertices $v(1) v(2) \cdots v(n)$ sush that $v(i)$ and $v(i+1)$ are adjacent in $C$ for $1 \leq i<n$. We can improve upon (10) by using the fact that a general connected graph can be decomposed into a tree of ncntrivial stars connected at cut vertices. Morcover, for hamiitonian connected graphs $(h(C)>0)$, sint a tree must "lways be a linear chrir. Thur, if we define the geucrating functions

$$
\begin{gathered}
\mathrm{G}(x)=\sum_{n=1}^{\infty} Z_{n} x^{n} \\
\mathbf{z}(x)=\sum_{\substack{\text { nonlrivial } \\
\text { stars }}} \frac{(-1)^{c(S)-\nu(S)+1}}{\nu(S) !} n(S)\left(\frac{1}{\tau(S)}\right)^{D / 2} x^{\nu(S)-1}
\end{gathered}
$$

then,

$$
\begin{aligned}
G(x) & =\sum_{k=1}^{\infty} z^{k} \\
& =\frac{z(x)}{1-z(x)} .
\end{aligned}
$$

To understand (11), consider a particular sequence of nontrivial hamiltonian stars $S_{1}, \ldots, S_{k}$ and choose hamiltonian paths for each one with beginning and ending vertices $\left(v_{1}, u_{1}\right), \ldots,\left(v_{k}, u_{k}\right)$. In order that $C=$ $S_{1} S_{2} \cdots S_{k}$ has a hamiltonian path beginning at $v_{1}$ and ending at $u_{k}$ the stars waust be glued together in a unique way; namely at the cut vertices $u_{1}=v_{2}, u_{2}=v_{3}, \ldots, u_{k-1}=v_{k}$. Morovver, it is also clear that $\tau(C)=$ $\tau\left(S_{1}\right) r\left(S_{2}\right) \cdots r\left(S_{k}\right)$ irrespective of the details of the decomposition. Finally, one can check that the counting of vertices and excess edges (minus signs) is correct. 
The series $\mathbf{z}(x)$ begins:

$$
z(x)=x-\left(\frac{1}{3}\right)^{D / 2} x^{2}+\left[-\left(\frac{1}{4}\right)^{D / 2}+3\left(\frac{1}{8}\right)^{D / 2}-\left(\frac{1}{16}\right)^{D / 2}\right] x^{3}+\cdots
$$

Values of $h(C)$ have been included in Table 1.

We again observe that the formal $D=0$ limit is easily evaluated. Considering (9) with $D=0$ we see that $Z_{n}=0$ for $n \geq 2$. Thus,

$$
\begin{array}{ll}
\mathrm{G}(x)=x & (D=0) \\
\mathrm{z}(x)=\frac{x}{1+x} . &
\end{array}
$$

\section{Conclusion}

A particularily interesting question concerns the nature of the singularities of the generating functions discussed above as the dimensionality is varied. If we formally set $D=\infty$, the singularities are simple poles:

$$
\begin{aligned}
& S(x)=\frac{1}{1-\frac{1}{2} x} \\
& \mathrm{G}(x)=\frac{x}{1-x} \\
& (D=\infty)
\end{aligned}
$$

This result is not unexpected and indeed the dominant singularity is believed to remain a simple pole provided $D>D_{c}$. The value of the critical dimension depends on the particular problem and it is believed that $D_{c}=6$ for percolation $^{6}$, and $D_{c}=4$ for self-avoiding walks ${ }^{7}$. Evidence of such a critical dimensionality might be obtained from numerical work with the scries expansions for $S(x)^{-1}$ and $z(x)$. 


\section{Acknowledgments}

This work was supported by the Director, Oflice of Energy Research, Ollice of High Euergy and Nuclear Physics, Division of High Energy Physics of the U. S. Department of Euergy under Contract DE-AC03-76SF00098.

\section{Appendix}

Proof of Lemma 1. We will use induction on $\nu(C)$. There is exactly one simple connected graph with two vertices, $C_{2}$. If we take $v$ to be either one of the two vertices then the nuclei that contain $v$ are $v$ itself and $C_{2}$ giving $1-1=0$ in (8). Now suppose the statement holds for all $C$ with $\nu(C)=2,3, \ldots, n-1$ for some $n \geq 3$. Let $C_{n}$ be any simple connected graph with $\nu\left(C_{n}\right)=n$ and let $\{v, u\} \in C_{n}$ be any two vertices connected by an cdge $e \in C_{n}$. The proof will proceed by decomposing $\eta_{v}$, the set of nuclci $N \subseteq C_{n}$ that contain $v$. We begin by defining the sets

$$
\begin{aligned}
& \eta_{1}=\left\{N \in \eta_{v} \mid e \notin N \text { and } u \in N\right\} \\
& \eta_{2}=\left\{N+e \mid N \in \eta_{1}\right\} .
\end{aligned}
$$

Clearly, $\eta_{2} \subseteq \eta_{v}$ and moreover, $\eta_{1} \cap \eta_{2}=\emptyset$. Thus,

$$
\begin{aligned}
\sum_{N \in \eta_{1} \cup \eta_{2}}(-1)^{\epsilon(N)} & =\sum_{N \in \eta_{1}}(-1)^{\epsilon(N)}+\sum_{N \in \eta_{1}}(-1)^{\epsilon(N)+1} \\
& =0 .
\end{aligned}
$$

The set of nuclei in the complement, $\eta_{v}-\left(\eta_{1} \cup \eta_{2}\right)$, is decomposed further:

$$
\begin{aligned}
& \eta_{3}=\left\{N \in \eta_{v} \mid u \notin N\right\} \\
& \eta_{4}=\left\{N \in \eta_{v}-\eta_{2} \mid e \in N\right\}
\end{aligned}
$$


For $N \in \eta_{4}$, suppose that $N-e$ is a nucleus. Then, $(N-e)+e=N \in$ $\eta_{2}$, a contradiction so $N-e$ cannot be a nucleus. This requires that $N-e$ consist of two components, $V(N)$ containing $v$ and $U(N)$ containing $u$. Let $v(N)$ be the vertex set of $V(N)$ and denote by $n(u)$ the set of vertices of $C_{n}$ adjaccnt to $u$.

We are now prepared to perform a decomposition of $\eta_{4}$ :

$$
\eta_{5}=\left\{N \in \eta_{4} \mid n(u) \subseteq v(N)\right\}
$$

It is clear that for $N \in \eta_{5}, U(N)=u$ and $u$ is adjacent only to $v$ in $N$. By deleting both $u$ and $e$ from these nuclei we have the following set:

$$
\eta_{6}=\left\{N-u-e \mid N \in \eta_{5}\right\}
$$

It is easily checked that $\eta_{3} \subseteq \eta_{3}$ and $\eta_{3} \subseteq \eta_{0}$ so that $\eta_{3}=\eta_{8}$. This establishes a one-onc correspondence between $\eta_{3}$ and $\eta_{5}$; the nuclei of $\eta_{5}$ having one additional edge. The sum over nuclei $N \in \eta_{3} \cup \eta_{5}$ thus vanishes in the manner of (12).

Finally, we consider the remaining nuclei $N \in \eta_{4}-\eta_{5}$. Deflne:

$$
\begin{aligned}
V & =\left\{V(N) \mid N \in \eta_{4}-\eta_{5}\right\} \\
\eta_{V} & =\left\{N \in \eta_{4}-\eta_{5} \mid V(N)=V\right\}
\end{aligned}
$$

For a particular $V \in V$, it is readily verified that $C_{n}-V$ breaks up into a set of components $\left\{w_{1}, \ldots, w_{k}, C_{u}(V)\right\}$ where $w_{1}, \ldots, w_{k}$ are isolated vertices and $C_{u}(V)$ is a nontrivial graph containing $u$. Moreover, it is also easily checked that the possible $U(N)$ for $N \in \eta_{V}$ are precisely the suclei of $C_{u}(V)$ that contain $u$. Since $v \notin C_{u}(V), 2 \leq \nu\left(C_{u}(V)\right)<n$ and we can apply iuduction: 


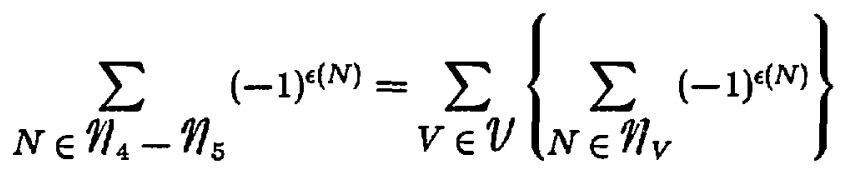

$$
\begin{aligned}
& =\sum_{V \in U}(-1)^{\epsilon(V)+1}\left\{\begin{array}{l}
\left.\sum_{\left\{N \subseteq C_{u}^{n u c l e i}(V) \mid u \in N\right\}}(-1)^{\epsilon(N)}\right\} \\
\}
\end{array}\right\} \\
& =0
\end{aligned}
$$




\section{References}

(1) G. Leibbrandt Rev. Mod. Phys. 47849 (1975)

(2) M. E. Fisher, D. S. Gaunt Phys. Rev. 133 A224 (1964)

M. F. Sykes, D. S. Gaunt, H. Ruskin J. Phys. A 91899 (1976)

(3) B. K. Kirchhoff Annalen d. Physik und Chemie 72497 (1847)

(4) G. E. Uhlenbeck, G. W. Ford In "Studies in Statistical Mechanics" Vol.1 p.182 (de Boer, Uhlenbeck eds.) North-Holland Publishing Co. Amsterdam (1962)

(5) D. Staulfer Phys. Rep. 54 no.l (1979)

(6) A. B. Harris, 'T. C. Lubensky, W. K. Holcomb, C. Dasgupta Phys. Rev. Lett. 35327 (1975)

(7) P. G. DeGennes Phys. Lett. 38A 339(1972) 


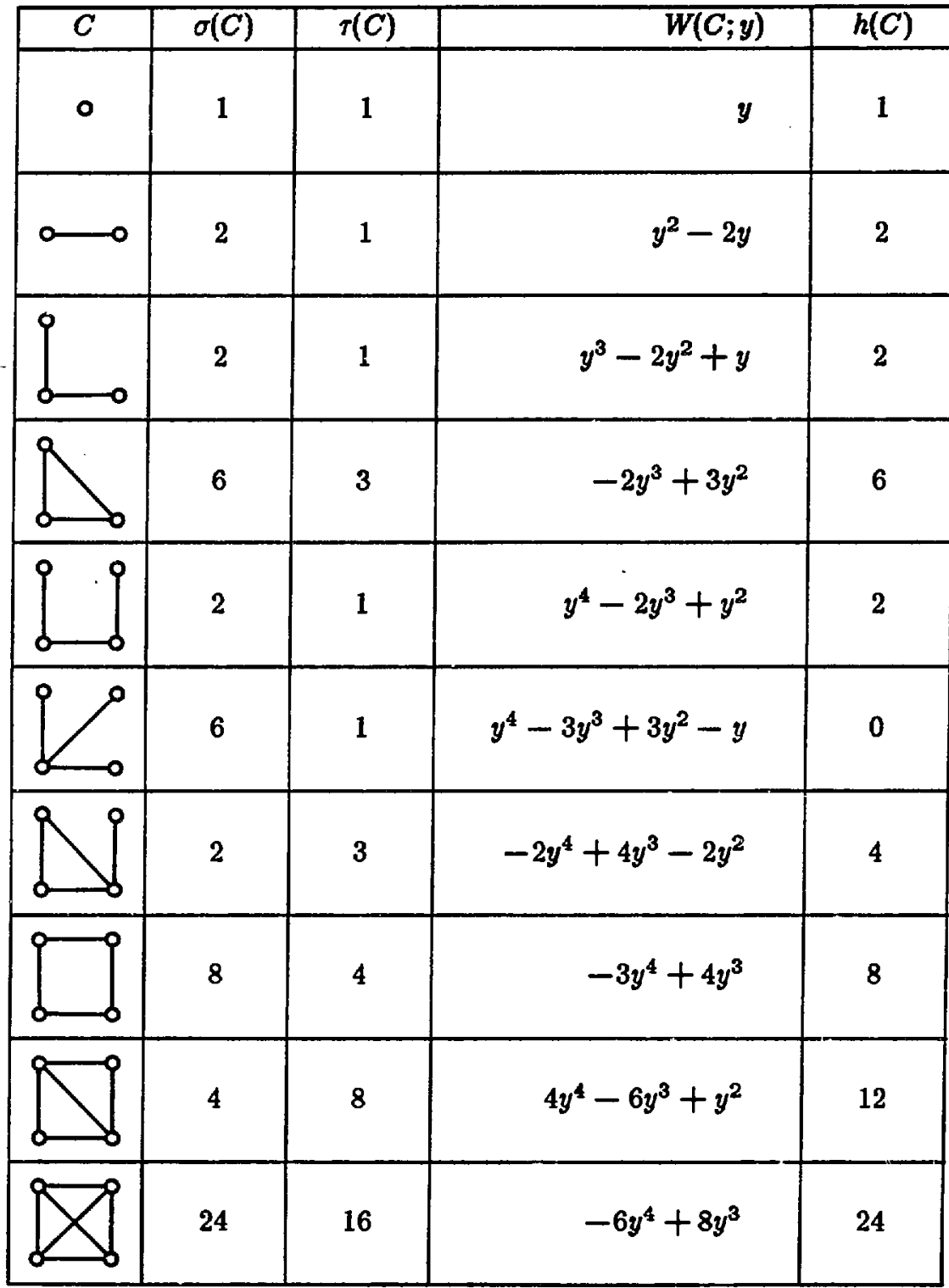

Table 1 
THE EQUILIBRIUM SHAPE OF CRYSTAL EDGES 


\section{Introduction}

It is well known that the large scale equilibrium shape of crystal surfaces is best characterized by facets. A realistic facet, however, is never infinite in extent and must meet other facets at finite intersection angles to form edges. Consequently, edges are a generic feature of the crystal surface the properties of which we would like to know.

For the simple-cubic crystal structure, a statistical mechanical model of the edge is provided by the partition function

$$
Z_{N}(n, m ; \beta)=\sum_{\left\{y_{x z}\right\}} \exp \left(-\beta \sum_{x, z}\left|y_{x z}-y_{x z+1}\right|\right)
$$

where the sum is over all the $n \times N$ sets of integer variables $\left\{y_{x z}\right\}$ that satisfy the constraint:

$$
0 \leq y_{1 z} \leq y_{2 z} \leq \cdots \leq y_{n z} \leq m \quad z=1, \ldots, N
$$

The Boltzmann factor of (1a) is precisely that of the usual SOS model for the special case that the height variables $y_{x z}$ are monotonic in the $x$-direction as implied by (1b). Another way of describing the interface as it is cut by a plane of constant $z$ is in terms of a lattice path taking $n$ steps in the $+x$-direction and $m$ steps in the $+y$-direction. Using this interpretation the Boltzmann weight of (1a) is just the sum of positive areas between consecutive lattice paths. Moreover, it obviously follows that $Z_{N}(n, m ; \beta)=Z_{N}(m, n ; \beta)$.

The free energy appropriate to (1) is given by $(L=n+m)$ :

$$
\lim _{\substack{N \rightarrow \infty \\ L \rightarrow \infty}}(N L)^{-1} \log Z_{N}(\rho L,(1-\rho) L ; \beta)=-F(\rho, \beta)
$$

Fere $\rho$ is the density of steps in the $+x$-direction. As $\rho \rightarrow 0(1)$ we approach the facet having $x=$ const ( $y=$ const) . 
THE EQUILIBRIUM SHAPE OF CRYSTAL EDGES 


\section{Introduction}

It is well known that the large scale equilibrium shape of crystal surfaces is best characterized by facets. A realistic facet, however, is never inflnite in extent and must meet other facets at finite intersection angles to form edges. Consequently, edges are a generic feature of the crystal surface the properties of which we would like to know.

For the simple-cubic crystal structure, a statistical mechanical model of the edge is provided by the partition function

$$
Z_{N}(n, m ; \beta)=\sum_{\left\{y_{x z}\right\}} \exp \left(-\beta \sum_{x, z}\left|y_{x z}-y_{x z+1}\right|\right)
$$

where the sum is over all the $n \times N$ sets of integer variables $\left\{y_{x: 3}\right\}$ that satisfy the constraint:

$$
0 \leq y_{1 z} \leq y_{2 z} \leq \cdots \leq y_{n z} \leq m \quad z=1, \ldots, N .
$$

The Boltzmann factor of (1a) is precisely that of the usual SOS model for the special case that the height variables $y_{x z}$ are monotonic in the $x$-direction as implied by (1b). Another way of describing the interface as it is cut by a plane of constant $z$ is in terms of a lattice path taking $n$ steps in the $+x$-direction and $m$ steps in the $+y$-direction. Using this interpretation the Boltzmann weight of (1a) is just the sum of positive areas between consecutive lattice paths. Moreover, it obviously follows that $Z_{N}(n, m ; \beta)=Z_{N}(m, n ; \beta)$.

The free energy appropriate to (1) is given by $(L=n+m)$ :

$$
\lim _{\substack{N \rightarrow \infty \\ L \rightarrow \infty}}(N L)^{-1} \log Z_{N}(\rho L,(1-\rho) L ; \beta)=-F(\rho, \beta) .
$$

Here $\rho$ is the density of steps in the $+x$-direction. As $\rho \rightarrow 0(1)$ we approach the facet having $x=$ const $(y=$ const) . 
The equilibrium shape of the crystal edge is obtained by the Wulff construction ${ }^{1}$. In order to proceed we have to be able to count the number of atoms in the crystal phase. We do this separately for each layer of constant $z$ and begin by choosing a completely filled quadrant of atoms as a reference configuration. As pointed out above, such an edge can be represented as a semi-infinite sequence of $+y$-steps followed by a semi-infinite sequence of $+x$-steps. For convenience, we consider each $x$-step as a particle and each $y$-step as the absence of a particle on a linear lattice. The reference configuration has all the sites to the right of the origin occupied and the remaining ones empty. Other conflgurations can be generated hy moving a finite number of the already existing particles. The statement of particle conservation can be expressed as

$$
0=\sum_{\xi>0}\left(n_{\xi}-1\right)+\sum_{\xi \leq 0} n_{\xi}
$$

where $\xi$ is the coordinate along the linear lattice and $n_{\xi}$ is the occupation number ( 0 or 1 ) of site $\xi$ (see figure 1).

To understand the general situation it is enough to follow the consequences of moving a single particle by one lattice unit. One discovers that a motion in the positive(negative) $\xi$-direction corresponds to the creation(annihilation) of a crystal atom at the interface. The number of atoms removed from the filled quadrant can thus be written as

$$
\mathcal{N}=-\sum_{\xi>0} \xi\left(n_{\xi}-1\right)-\sum_{\xi \leq 0} \xi n_{\xi} .
$$

When $\mathcal{N}$ is large and the conflguration is more appropriately described by a density of particles $\rho(\xi)$, we use the continuum forms of (3) and (4):

$$
\begin{gathered}
0=\int_{0}^{\infty}(\rho(\xi)-1) d \xi+\int_{-\infty}^{0} \rho(\xi) d \xi \\
N=-\int_{0}^{\infty} \xi(\rho(\xi)-1) d \xi-\int_{-\infty}^{0} \xi \rho(\xi) d \xi .
\end{gathered}
$$


We can now apply the Wulf construction and obtain the functional

$$
W[\rho]=\int_{-\infty}^{\infty}\left(\lambda\left(\xi-\xi_{0}\right) \rho(\xi)-F(\rho, \beta)\right) d \xi
$$

where the Lagrange multipliers $\lambda \xi_{0}$ and $\lambda$ apply to constraints ( $\left.3^{\prime}\right)$ and (4') respectively. The equilibrium shape can now be found by maximizing $F i[\rho]$ subject to the conditions:

$$
\begin{aligned}
0 & \leq \rho(\xi) \leq 1 \\
\lim _{\xi \rightarrow-\infty} \rho(\xi) & =0 \quad \lim _{\xi \rightarrow \infty} \rho(\xi)=1 .
\end{aligned}
$$

The functional $W[\rho]$ is extremized for the choice

$$
\frac{\partial}{\partial \rho} F(\rho, \beta)=\lambda\left(\xi-\xi_{0}\right) \equiv t
$$

which can be inverted (locally) to yield $\rho$ as a function of $\xi$. However, we will see in subsequent calculations that for $\beta>0$

$$
\begin{gathered}
\frac{\partial}{\partial \rho} F(0, \beta)=-t_{0} \quad \frac{\partial}{\partial \rho} F(1, \beta)=t_{0} \\
-t_{0}<\frac{\partial}{\partial \rho} F(\rho, \beta)<t_{0} \quad(0<\rho<1)
\end{gathered}
$$

where $t_{0}>0$ is flnite. This means that in poneral,

$$
\rho(t)=\left\{\begin{array}{lc}
0 & \hat{\vdots}<-t_{0} \\
\left(\partial_{\rho} F\right)^{-1}(t) & -t_{0}<t<t_{0} \\
1 & t_{0}<t .
\end{array}\right.
$$

The parameters $\xi_{0}$ and $\lambda$ are related to the position and scale of the edge respectively; their values are determined by equations ( $\left.3^{\prime}\right)$ 
and $\left(4^{\prime}\right)$. The coordinate $\xi$ has the geometrical interpretation of being measured along an axis rotated $45^{\circ}$ with respect to the original $x$ and $y$ axes of the crystal (see figure 1). If in some range from $\xi$ to $\xi+\Delta \xi$ the lattice path makes $n$ steps in the $+x$-direction and $m$ steps in the $+y$-direction, the change in position $\Delta \eta$, perpendicular to the $\xi$-axis is just $m-n$. Consequently,

$$
\frac{\Delta \eta}{\Delta \xi}=\frac{m-n}{n+m}=1-2 \rho
$$

Finally, upon substituting the rescaled variables $t=\lambda \xi$ and $s=\lambda \eta$, the edge profile is given by the expression

$$
s(t)=\int_{0}^{t}(1-2 \rho(u)) d u+\text { const } .
$$

In the following sections the free energy (2) will be calculated in the limits $\beta \rightarrow \infty, \beta \rightarrow 0$, and $\rho \rightarrow 0$. These results can then be used to obtain the low and high temperature limits of the edge profile as well as the behavior in the region where the surface joins a facet.

\section{Low Temperature Expansion}

The partition function (1) can in principle be expressed in terms of a transfer matrix $M$ as

$$
Z_{N}(n, m ; \beta)=\operatorname{tr} M^{N}
$$

The elements of $M$ are simple, however, only in the limit $\beta \rightarrow \infty$ when they can be ezpanded in powers of $e^{-\beta}$. If we let $L=n+m$ then the 'states' appropriate to $M$, as discussed in section 1 , are the conflgurations 
of $n$ particles on a linear lattice of $L$ sites. The matrix element between two such states is unity when the states are the same and $e^{-\theta}$ if the states differ by the displacement of one particle by one unit. If we neglect the other matrix elements which are $O\left(e^{-2 \beta}\right)$, then $M$ is naturally written in the form

$$
M=1+e^{-\beta} \sum_{i=1}^{L-1}\left(a_{i+1}^{\dagger} a_{i}+a_{i}^{\dagger} a_{i+1}\right)+O\left(e^{-2 \beta}\right)
$$

The operators $a^{\dagger}$ and $a$ are the usual bosonic creation and annihilation operators with the additional properties

$$
\left(a_{i}^{\dagger}\right)^{2}=\left(a_{i}\right)^{2}=0
$$

This is necessary since we require that $M$ acting on a state never produce a state with two particles occupying the same site.

When $M$ is written as the exponential of a hamiltonian the free energy (2) can be expressed in terms of the lowest $n$-particle energy eigenvalue $E(L, n)$ :

$$
\begin{aligned}
M & =\exp \left(-H_{1}+O\left(e^{-2 \beta}\right)\right) \\
H_{1} & =-e^{-\beta} \sum_{i=1}^{L-1}\left(a_{i+1}^{\dagger} a_{i}+a_{i}^{\dagger} a_{i+1}\right) \\
F(\rho, \beta) & =\lim _{L \rightarrow \infty} L^{-1} E(L, \rho L) .
\end{aligned}
$$

By use of the Jordan-Wigner transformation ${ }^{2}$ the operators introduced above can be written in terms of fermionic operators with the result that the operators in $H_{1}$ are now interpreted as fermionic. Since we have effectively hard-wall boundary conditions, the hamiltonian is diagonalized 
by the canonical transformation

$$
\begin{gathered}
a_{i}^{(\dagger)}=\sqrt{\xi} \sum_{k=1}^{L} \sin \left(\frac{z^{i}}{t+}\right) b(t) \\
H_{1}=-e^{-\beta} \sum_{k=1}^{L} \epsilon(k) b_{k}^{\dagger} b_{k} \quad \epsilon(k)=2 \cos \left(\frac{\pi k}{L+1}\right)
\end{gathered}
$$

with ground state eigenvector and energy given by:

$$
\begin{aligned}
\Psi_{0} & =\prod_{k=1}^{n} b_{k}^{\dagger}|0\rangle \\
E(L, n) & =-e^{-\beta} \sum_{k=1}^{n} \epsilon(k)+O\left(e^{-2 \beta}\right) \\
F(\rho, \beta) & =-\frac{2}{\pi} \sin (\pi \rho) e^{-\beta}+O\left(e^{-2 \beta}\right) .
\end{aligned}
$$

We see that the form of the free energy as a function of $\rho$ agrees with the claims made in section 1 . Using equations (5) and (6) we have

$$
\begin{aligned}
& t_{0}=2 e^{-\beta} \\
& \rho(t)=\frac{1}{\pi} \cos ^{-1}\left(\frac{-t}{t_{0}}\right) \quad\left(-t_{0}<t \cdot<t_{0}\right)
\end{aligned}
$$

with the edge profile given by (7):

$$
s(t)=\left\{\begin{array}{lr}
t & t<-t_{0} \\
t-\frac{2}{\pi}\left(t \cos ^{-1}\left(\frac{-t}{t_{0}}\right)+\sqrt{t_{0}^{2}-t^{2}}\right) & -t_{0}<t<t_{0} \\
-t & t_{0}<t .
\end{array}\right.
$$

It is apparent from (9) that the density $\rho(t)$ has square-root singularities at $t= \pm t_{0}$ (see figure 2). Since $s(t)$ is essentially the integral 
of $\rho(t)$ this implies that the edge profile joins the facet with a $t^{3 / 2}$ behavior. The square-root singularities of $\rho$ can be traced to the fact that the present form of the free energy satisfles

$$
\frac{\partial^{2}}{\partial \rho^{2}} F(0, \beta)=0 \quad \frac{\partial^{2}}{\partial \rho^{2}} F(1, \beta)=0
$$

As will be shown later, the above holds for all finite temperatures so that the $t^{3 / 2}$ behavior of the surface near the facet follows in general.

To illustrate the nature of the expansion we will also calculate the $O\left(e^{-2 \beta}\right)$ term of the free energy. It is flrst necessary to extend the transfer matrix (8) to include operators that generate two units of particle displacement. Most of these operators appear in the product $\frac{1}{2} H_{1}^{2}$ where $H_{1}$ is the $O\left(e^{-\beta}\right)$ piece of the hamiltonian. Being the product of two 'hopping' operators $\left(a_{i \pm 1}^{\dagger} a_{i}\right)\left(a_{j \pm 1}^{\dagger} a_{j}\right)$, these terms are correct except possibly when their subscripts overlap. To correct for these possible mistakes, we subtract out all the overlapping products and add in the correct terms. The latter are

$$
\begin{aligned}
& a_{i}^{\dagger} a_{i-1} a_{i+1}^{\dagger} a_{i}+\text { h.c. } \\
& a_{i+1}^{\dagger} a_{i} a_{i}^{\dagger} a_{i-1}+\text { h.c. }
\end{aligned}
$$

where (11) moves two particles each by one unit while (12) moves a single particle through two units. Following the above strategy, the transfer matrix can by written as 


$$
\begin{aligned}
& M=1-H_{1}+\frac{1}{2} H_{1}^{2} \\
& -e^{-2 \beta}\left\{\frac{1}{2} \sum_{i=2}^{L-1}\left[\left(a_{i}^{\dagger} a_{i-1}\right)\left(a_{i+1}^{\dagger} a_{i}\right)+\left(a_{i+1}^{\dagger} a_{i}\right)\left(a_{i}^{\dagger} a_{i-1}\right)+\text { h.c. }\right]\right. \\
& \left.+\frac{1}{2} \sum_{i=1}^{L-1}\left[\left(a_{i+1}^{\dagger} a_{i}\right)\left(a_{i}^{\dagger} a_{i+1}\right)+\left(a_{i}^{\dagger} a_{i+1}\right)\left(a_{i+1}^{\dagger} a_{i}\right)\right]\right\} \\
& +e^{-2 \beta} \sum_{i=2}^{L-1}\left[a_{i}^{\dagger} a_{i-1} a_{i+1}^{\dagger} a_{i}+a_{i+1}^{\dagger} a_{i} a_{i}^{\dagger} a_{i-i}+\text { h.c. }\right] \\
& +O\left(e^{-3 \beta}\right) .
\end{aligned}
$$

The Jordan-Wigner transformation replaces the pairs $a_{i 1}^{\dagger} a_{i}$ by corresponding fermionic operators so that once again we may interpret all the operators as fermionic. Taking the logarithm of $M$ we obtain the hamiltonian

$$
\begin{aligned}
& H=H_{1}+H_{2}+O\left(e^{-3 \beta}\right) \\
& H_{2}=e^{-2 \beta}\left\{\sum_{i=2}^{L-1}\left[a_{i+1}^{\dagger}\left(a_{i}^{\dagger} a_{i}-\frac{1}{2}\right) a_{i-1}+\text { h.c. }\right]\right. \\
&\left.\sum_{i=1}^{L-1} a_{i+1}^{\dagger} a_{i}^{\dagger} a_{i+1} a_{i}+n\right\} .
\end{aligned}
$$

In one of the simpliflcations the number operator was replaced by $n$, the number of particles. It is now a straightforward problem to evaluate the correction to the ground state energy by taking the expectation value of $\mathrm{H}_{2}$ in the zeroth order ground state. This part of the calculation is relatively unenlightening so we merely give the final result: 


$$
\begin{aligned}
F(\rho, \beta)= & -\frac{2}{\pi} \sin (\pi \rho) e^{-\beta} \\
& +\left[\rho(1-\rho)+\frac{2 \rho-1}{2 \pi} \sin (2 \pi \rho)-\frac{1}{\pi^{2}} \sin ^{2}(\pi \rho)\right] e^{-2 \beta} \\
& +O\left(e^{-3 \beta}\right)
\end{aligned}
$$

As a simple check we note that the correction is symmetric about $\rho=\frac{1}{2}$. it can also be verifled that this term satisfies equation (10).

\section{High Temperature Limit}

We will concentrate again on the $\xi$ and $\eta$ coordinate system introduced earlier. The set of lattice paths or cross-sections of the interface may be thought of as random walks $\eta_{1}(\xi), \ldots, \eta_{N}(\xi)$ with $\xi$ as a common 'time' parameter. For each step in time the particle positions $\eta_{i}(\xi)$ change by \pm 1 with the Boltzmann weight acting as an attractive force between consecutive particles. In the limit $\beta \rightarrow 0$ the particles can drift very far apart so that over short periods of time the fluctuations in the positive area between two consecutive paths are unimportant. In other words, for periods of time that are in some sense small compared to the separation between consecutive particles, the random walks are free.

We have to be careful however, to remember the global constraint that during a time $\Delta \xi$ the random walk makes on the average exactly $\rho \Delta \xi$ steps in the $+x$-direction. This is evident from the boundary conditions (1a) of the partition function. However, for our fresent purposes it will be more convenient to let the boundaries be free while introducing activities $\rho$ and $1-\rho$ respectively for motion in the $+x$ and $+y$ directions. To 
recover the original partition function (1) we must then divide by

$$
\left[\rho^{n}(1-\rho)^{m}\right]^{N}
$$

which merely adds the constant

$$
\rho \log \rho+(1-\rho) \log (1-\rho)
$$

to the free energy.

Now suppoce that the particle positions are $\eta_{1}, \ldots, \eta_{N}$ at some initial time $\xi_{0}$ and $\eta_{1}^{\prime}, \ldots, \eta_{N}^{\prime}$ at some later time $\xi_{0}+\Delta \xi$. For $\Delta \xi \gg 1$ but fixed as $\beta \rightarrow 0$ we will be able to sum over the conflgurations at intermediate times and thereby obtain a transfer matrix. In this limit it will almost always be true that

$$
\left|\eta_{i}-\eta_{i}^{\prime}\right| \ll\left|\eta_{i}-\eta_{i \pm 1}\right| \simeq\left|\eta_{i}^{\prime}-\eta_{i \pm 1}^{\prime}\right|
$$

so that the Boltzmann factor is relatively constant and can be taken outside the summation. In terms of the $\xi$ and $\eta$ coordinates we see from figure 1 that this factor is given by

$$
\exp \left\{-\frac{1}{2} \beta \Delta \xi \sum_{i=1}^{N-1}\left|\eta_{i}-\eta_{i+1}\right|\right\}
$$

What remains is just the sum over $N$ independent random walks having specified endpoints and the activity factors discussed above ( $\Delta \eta_{i}=$ $\left.\eta_{i}^{\prime}-\eta_{i}\right):$

$$
\prod_{i=1}^{N}\left(\begin{array}{c}
\Delta \xi \\
\frac{1}{2}\left(\Delta \xi-\Delta \eta_{j}\right)
\end{array}\right) \rho^{\frac{1}{2}\left(\Delta \xi-\Delta \eta_{i}\right)}(1-\rho)^{\frac{1}{2}\left(\Delta \xi+\Delta \eta_{i}\right)} .
$$

Since $\Delta \xi>1$, it can be shown that each term in the product is strongly peaked at $\Delta \eta_{i}=(1-2 \rho) \Delta \xi$. If we change from the $\eta$-coordinates to the 
new set $z_{i}=\eta_{i}-(1-2 \rho) \xi$, then (16) takes the asymptotic form:

$$
\prod_{i=1}^{N} \frac{1}{\sqrt{8 \pi \rho(1-\rho) \Delta \xi}} \exp \left\{-\frac{\left(z_{i}^{\prime}-z_{i}\right)^{2}}{8 \rho(1-\rho) \Delta \xi}\right\} \text {. }
$$

From the Gaussian factor in (17) it is clear that the $z_{i}$ are effectively continuum variables. Changing to the $z_{i}$ variables in (15) and taking the product with (17) we end up with the transfer matrix:

$$
\begin{aligned}
K\left(z, z^{\prime} ; \Delta \xi\right)=\left(\frac{1}{8 \pi \rho(1-\rho) \Delta \xi}\right)^{N / 2} \exp \{ & -\sum_{i=1}^{N} \frac{\left(z_{i}^{\prime}-z_{i}\right)^{2}}{8 \rho(1-\rho) \Delta \xi} \\
& \left.-\frac{1}{2} \beta \Delta \xi \sum_{i=1}^{N-1}\left|z_{i}-z_{i+1}\right|\right\} .
\end{aligned}
$$

This result is indistinguishable from the short-time kernel

$$
K\left(z, z^{\prime} ; t\right)=\lim _{t \rightarrow 0}\left\langle z^{\prime}\left|\exp \left(-t H_{N}\right)\right| z\right\rangle
$$

with continuum hamiltonian

$$
H_{N}=2 \rho(1-\rho) \sum_{i=1}^{N} p_{i}^{2}+\frac{1}{2} \beta \sum_{i=1}^{N-1}\left|z_{i}-z_{i+1}\right|
$$

where $t=\Delta \xi$.

The validity of this derivation depended on having the mean separation between consecutive particles be large. This is a statement about the ground state wavefunction that we can now test. Suppose the mean separation is of order $l$, then $p_{i}^{2}$ is of order $l^{-2}$. Since the kinetic and potential parts of the hamiltonian have the same order of magnitude in the ground state we have that

$$
\frac{\rho(1-\rho)}{l^{2}} \sim \beta i
$$


or

$$
\operatorname{l\sim }\left(\frac{\rho(1-\rho)}{\beta}\right)^{1 / 3}>1
$$

Since this breaks down for $\rho \rightarrow 0$ and $\rho \rightarrow 1$, the present approximation cannot give the behavior of the surface near the crystal facets.

Recalling that the free energy (2) was deflned per unit of $\xi$, we see that this is just $E_{N} / N$ where $E_{N}$ is the ground state energy of $H_{N}$. The $\beta$ and $\rho$ dependence of $E_{N}$ can be made explicit by the rescaling

$$
\begin{gathered}
z=2\left(\frac{\rho(1-\rho)}{\beta}\right)^{1 / 3} x \\
H_{N}=\left(\rho(1-\rho) \beta^{2}\right)^{1 / 3} \bar{H}_{N} \\
\bar{H}_{N}=\frac{1}{2} \sum_{i=1}^{N} p_{i}^{2}+\sum_{i=1}^{N-1}\left|x_{i}-x_{i+1}\right|
\end{gathered}
$$

If the ground state energy of $\bar{H}_{N}$ is $\bar{E}_{N}$ and

$$
e_{0}=\lim _{N \rightarrow \infty} N^{-1} \bar{E}_{N}
$$

then, remembering to include (14), our final result is:

$$
\begin{aligned}
F(\rho, \beta)=\rho \log \rho & +(1-\rho) \log (1-\rho) \\
& +e_{0}\left(\rho(1-\rho) \beta^{2}\right)^{1 / 3} \quad \beta<\rho(1-\rho) .
\end{aligned}
$$

The exact value of $e_{0}$ is not known but it is easy to obtain the variational bound

$$
e_{0}<1.0188
$$


using the trial wavefunction

$$
\Psi_{0}=\prod_{i=1}^{N-1} \Phi\left(x_{i}-x_{i+1}\right)
$$

\section{Low Density Expansion}

We will consider once again the transfer matrix $M$ introduced in section 2. However, rather than derive an approximate hamiltonian as was dune for the low temperature expansion, we will try to compute the largest eigenvalue of $M$ directly. In terms of the original $y_{c z}$ variables, the eigenvalue equation takes the form

$$
\Lambda_{n m} \Psi\left(y_{1}, \ldots, y_{n}\right)=\sum_{y^{\prime} \in R} \exp \left(-\beta \sum_{i=1}^{n}\left|y_{i}-y_{i}^{\prime}\right|\right) \Psi\left(y_{1}^{\prime}, \ldots, y_{n}^{\prime}\right)
$$

where by $y^{\prime} \in R$ we mean that the sum is over the region

$$
R: \quad 0 \leq y_{1}^{\prime} \leq \cdots \leq y_{n}^{\prime} \leq m \text {. }
$$

The free energy is now given by

$$
\lim _{L \rightarrow \infty} L^{-1} \log \Lambda_{\rho L}(1-\rho) L=-F(\rho, \beta)
$$

We can view (18) as a kernel for $n$ particles moving on a linear lattice of $m+1$ sites. At low particle density the separations $\left|y_{i}-y_{i}^{\prime}\right|$ are of the order $\beta^{-1}$ so that there is little interaction among the particles when $\beta^{-1}<m / n$ or $\rho \ll \beta$. In this limit (18) approaches the diffusion kernel for $n$ particles that are prevented from moving through each other. 
The eigenvalue equation (18) defines the eigenfunction $\Psi(y)$ also when the point $y=\left(y_{1}, \ldots, y_{n}\right)$ lies outside the region $R$. It is therefore valid to $w$ rite equations for $\Psi(y)$ that sample points outside this region. One such equation involves the second order difference operator

$$
\nabla f(x) \equiv \cosh \beta f(x)-\frac{1}{2}[f(x+1)+f(x-1)]
$$

with the property:

$$
\nabla \exp \left(-\beta\left|x-x^{\prime}\right|\right)=\sinh \beta \delta_{x x^{\prime}}
$$

If we apply this operator $n$ times on (18) we obtain the equation

$$
\Lambda_{n m} \nabla_{1} \cdots \nabla_{n} \Psi(y)=(\sinh \beta)^{n} \theta(y) \Psi(y)
$$

where

$$
\theta(y)= \begin{cases}1 & y \in R \\ 0 & \text { otherwise }\end{cases}
$$

A different sort of equation using the first order difference operator

$$
\partial f(x) \equiv f(x+1)-f(x)
$$

follows from the identity:

$$
\begin{gathered}
\left.\left(\partial_{1}+1-e^{-\beta}\right)\left(\partial_{2}+1-e^{\beta}\right) \exp \left(-\beta\left|x_{1}-x_{1}^{\prime}\right|-\beta\left|x_{2}-x_{2}^{\prime}\right|\right)\right|_{x_{1}}=x_{2} \\
=0 \quad \text { for } x_{1}^{\prime} \leq x_{2}^{\prime} .
\end{gathered}
$$

Since the above inequality is satisfled by each pair of consecutive variables $y_{i}^{\prime} \leq y_{i+1}^{\prime}$ in the summation region $R$, we can derive from (18) the boundary conditions $(i=1, \ldots, n-1)$ :

$$
\left.\left(\partial_{i}+1-e^{-\beta}\right)\left(\partial_{i+1}+1-e^{\beta}\right) \Psi(y)\right|_{y_{i}=y_{i+1}}=0
$$


Equation (20) together with the set of boundary conditions (21) are reminiscent of some one-dimensional many-body problems that can be solved ezactly using the Bethe-ansatz method ${ }^{3}$. In particular, if we take the limit $\beta \rightarrow 0$ while still maintaining $\rho \ll \beta$, the product of the operators $\nabla$ in (21) exponentiates to give

$$
\exp \left(-\beta^{-2} \sum_{i=1}^{n} \frac{\partial^{2}}{\partial y_{i}^{2}}\right) \Psi(y)=\frac{1}{\Lambda_{n m}}\left(\frac{2}{\beta}\right)^{n} \theta(y) \Psi(y)
$$

since the momentum components of $\Psi(y)$ are of the order $\rho \ll \beta$. In the sam a limit the boundary conditions (21) become

$$
\left.\left(\frac{\partial}{\partial y_{i+1}}-\frac{\partial}{\partial y_{i}}-\beta\right) \Psi(y)\right|_{y_{i}=y_{i+1}}=0 \quad i=1, \ldots, n-1
$$

giving us the full set of equations that define the problem of the 'deltafunction gas' 4.

The exact equations (20) and (21) can in fact also be solved using the Bethe-ansatz. Unfortunately, however, this solution does not satisfy the original eigenvalue equation (18) except in the limit of ranishing density. In order to understand this rather remarkable failure it is perhaps instructive to consider in detail the two-body problem first.

Wheir the two particles are free to move on an infinite line of lattice sites we have to solve the equation

$$
\Lambda \Psi\left(y_{1}, y_{2}\right)=\sum_{y_{1}^{\prime} \leq y_{2}^{\prime}} \exp \left(-\beta\left|y_{1}-y_{1}^{\prime}\right|-\beta\left|y_{2}-y_{2}^{\prime}\right|\right) \Psi\left(y_{1}^{\prime}, y_{2}^{\prime}\right) .
$$

The center of mass motion can be eliminated using

$$
\Psi\left(y_{1}, y_{2}\right)=\exp \left(i k y_{1}\right) R\left(y_{2}-y_{1}\right)
$$

where now, in terms of the relative coordinate $r=y_{2}-y_{1} \geq 0$, the eigenvalue equation becomes:

$$
\Lambda R(\boldsymbol{r})=\sum_{\boldsymbol{r}^{\prime}=0}^{\infty} K\left(\boldsymbol{r}-\boldsymbol{r}^{\prime}\right) R\left(\boldsymbol{r}^{\prime}\right)
$$




$$
K(u)=K(-u)^{*}=\sum_{v=-\infty}^{\infty} \exp (-\beta|v|-\beta|u+v|-i k v)
$$

Equation (22) is of the kind that can be solved using the WienerHopf method. Since this method is explained at length elsewhere (see for example ref. 5) we will only give the final answer. Although the form of our wavefunction is exact, the constants that appear have been approximated for the case that $\Lambda$ is in the vicinity of the maximum eigenvalue. These wavefunctions are best characterized in terms of two small momentum values $p_{1}$ and $p_{2}$ :

$$
\begin{array}{r}
\Psi\left(y_{1}, y_{2}\right)=\exp \left(i p_{1} y_{1}+i p_{2} y_{2}\right)-\exp (i \phi) \exp \left(i p_{2} y_{1}+i p_{1} y_{2}\right) \\
+A \exp \left(i \frac{1}{2}\left(y_{1}+p_{2}\right)\left(y_{1}+y_{2}\right)-b\left(y_{2}-y_{1}\right)\right)
\end{array}
$$

where,

$$
\begin{aligned}
& \phi=c\left(p_{1}-p_{2}\right)+O\left(p^{2}\right) \\
& A=i \frac{\left(e^{-b}-e^{-\beta}\right)^{2}}{\left(1-e^{-b}\right)\left(1-e^{-\beta}\right)^{2}}\left(p_{1}-p_{2}\right)+O\left(p^{2}\right)
\end{aligned}
$$

and,

$$
\begin{gathered}
b=-\log \left(1-4 v\left(\sqrt{1+\frac{1}{2 v}}-1\right)\right)+O(p) \\
c=1+\sqrt{1+\frac{1}{v}}-\frac{1}{4 v}\left(\sqrt{1+\frac{1}{2 v}}-1\right)^{-1} \\
v=\left(\sinh \frac{\beta}{2}\right)^{2} .
\end{gathered}
$$

We see that the first two terms of the wavefunction (23) have exactly the Bethe-ansatz form with the phase shift given by $\phi$. However, there is also an expor.entially decaying term that describes a 'bound-state' 
piece of the warefunction. It is this term that spoils the Bethe-ansatz. One would also expect analogues of this term to arise in the general $n$-body problem. We can nevertheless make some progress by taking advantage of the fact that relative to the plane-wave terms, the bound-state amplitude is small:

$$
A \sim O(p) \sim O(\rho)
$$

The first term in a low density expansion would thus proceed along the Bethe-ansatz lines while pretending $A=0$. The question is then the following: to what order in momentum may we keep terms in the phase shift $\phi$ ? We conjecture that it is valid to retain the terms up to $O(p)$ but cannot provide a simple proof of this claim. Some evidence in favor will appear at the end of this section.

The boundary conditions implicit in (18) due to the endpoints of the lattice are not ideally suited to the present discussion. We therefore modify the original problem by wrapping the lattice into a circle of $\mathrm{m}$ points. This should not affect the thermodynamic limit and allows us to impose the periodic boundary conditions

$$
\Psi\left(y_{1}, \ldots, y_{n}\right)=\Psi\left(y_{2}, \ldots, y_{n}, m+y_{1}\right)
$$

One of the terms appearing in the wavefunction $\Psi$ is the product of $n$ plane waves:

$$
\exp \left(i p_{1} y_{1}+\cdots+i p_{n} y_{n}\right)
$$

By suitably normalizing $\Psi$ the coefficient of this term can be set equal to unity. Other terms in $\Psi$ obtained from (26) by permuting the momenta will have coefficients given by appropriate phases. For the permutation that shifts the momenta in an $n$-cycle the phase can be deduced trivially since by (25) the term (26) becomes

$$
\exp \left(i p_{n} m\right) \exp \left(i p_{n} y_{1}+i p_{1} y_{2}+\cdots+i p_{n-1} y_{n}\right)
$$


In the usual Bethe-ansatz problem one generaies the same permutation by $t$ l. e sequence of transpositions

$$
T_{2} \cdots T_{n-1} T_{n}
$$

$$
\begin{aligned}
T_{i}: \quad \exp \left(i p_{i-1} y_{i-1}+i p_{n} y_{i}\right) \rightarrow \\
-\exp \left(i \phi\left(p_{i-1}, p_{n}\right)\right) \exp \left(i p_{n} y_{i-1}+i p_{i-1} y_{i}\right)
\end{aligned}
$$

where the phase shifts $\phi$ are obtained directly from an equation of the type (21). In the present situation, however, we use the result (24) of the twobody problem. Equating the phase in (27) with the accumulated phase from (28) we end up with the set of equations $\left(N_{i}=\right.$ integer, $n=$ odd):

$$
\begin{aligned}
p_{i} m & =\sum_{j \neq i} \phi\left(p_{j}, p_{i}\right)+2 \pi N_{i} \\
& =-c n p_{i}+c \sum_{j} p_{j}+2 \pi N_{i} .
\end{aligned}
$$

A nontrivial ground state wavefunction is found by choosing a distinct set of momenta that satisfy (29) and maximize the eigenvalue $\Lambda_{n m}$. Recalling the action of the difference operator

$$
\nabla \exp (i p y)=(\cosh \beta-\cos p) \exp (i p y),
$$

the eigenvalue, according to (20), is just

$$
\Lambda_{n m}=\prod_{i=1}^{n}\left(\frac{\sinh \beta}{\cosh \beta-\cos p_{i}}\right) .
$$

From (as) 70 see illat (to this approsimation) the momenta are equally spaced. The maximum eigenvalue results when these are chcsen symmetrically about $p=0$ :

$$
p_{i}=\frac{2 \pi N_{i}}{m}\left(1-c \frac{n}{m}+O\left(\rho^{2}\right)\right)
$$




$$
-\frac{1}{2}(n-1) \leq N_{i} \leq \frac{1}{2}(n-1) .
$$

Using (19) we arrive at our final result:

$$
\begin{gathered}
F(\rho, \beta)=-\rho \log \left(\frac{\sinh \beta}{\cosh \beta-1}\right)+\frac{\pi}{6}^{2}\left(\frac{1}{\cosh \beta-1}\right)\left(\rho^{3}+2(1-c) \rho^{4}\right) \\
+O\left(\rho^{5}\right)
\end{gathered}
$$

The first term above is simply related to the free energy of a single particle on an infinite lattice, while the $O\left(\rho^{3}\right)$ term reflects the impenetrability of the two adjacent particles. These terms are insensitive io the precise nature of the interaction among the particles except tr 3 t these are short range and hard-core. The $O(p)$ dependence of the phase shift first appears in the $O\left(p^{4}\right)$ term of $(30)$ and required the solution of the two-body problem. Presumably the $O\left(\rho^{5}\right)$ tarm will involve the inclusion of three-body effects.

A useful check on our result (in particular the $O\left(\rho^{4}\right)$ term) follows from the observation that (30) and the low temperature result (13) have a common region of validity. Indeed, it can be verified that an expansion of the coefficients of (30) in powers of $e^{-\beta}$ agrees with the expansion of (13) in powers of $\rho$.

Finally, we observe that the $O\left(\rho^{2}\right)$ term vanishes for all values of the temperature, thus confirming (10) and our claims about the behavior of the surface near a facet.

After the completion of this work it was pointed out to the author that the $t^{3 / 2}$ behayior near the crystal facet has been discussed previously ${ }^{6}$. 


\section{Acknowledgments}

The author thanks D. S. Fisher for pointing out ref. 6, O. Alvarez and J. Carlson for TEXnical suggestions. This work was supported oy the Director, Offlce of Energy Research, Offlce of High Energy and Nuclear Physics, Division of High Energy Physics of the U. S. Department of Energy under Contract DE-AC03-76SF00098.

\section{References}

(1) see, e.g., L. D. Landan and E. M. Lifshitz "Statistical Physics" (Addison-Wesley, Reading, Mass., 1969) sec. 143

(2) see, e.g., F. H. Lieb, T. D. Schultz, and D. C. Mattis Ann. Phys. 16 $407(1961)$

(3) see, e.g., E. H. Lieb and D. C. Mattis "Mathematical Physics in One Dimension" (Academic Press, New York, 1966) ch. 6

(4) E. H. Lieb and W. Liniger Phys. Rev. 1301605 (1963)

(5) J. Mathews and R. L. Walker "Mathematical Methods of Physics" (W. A. Benjamin Inc., New York, 1970) p. 312

(6) C. Jayaprakash et. al. Phys. Rev. Lett. 502017 (1983)

\section{Figure Captions}

Fig. 1: Two lattice paths and their particle representations.

Fig. 2: The particle density in the low temperature limit. 


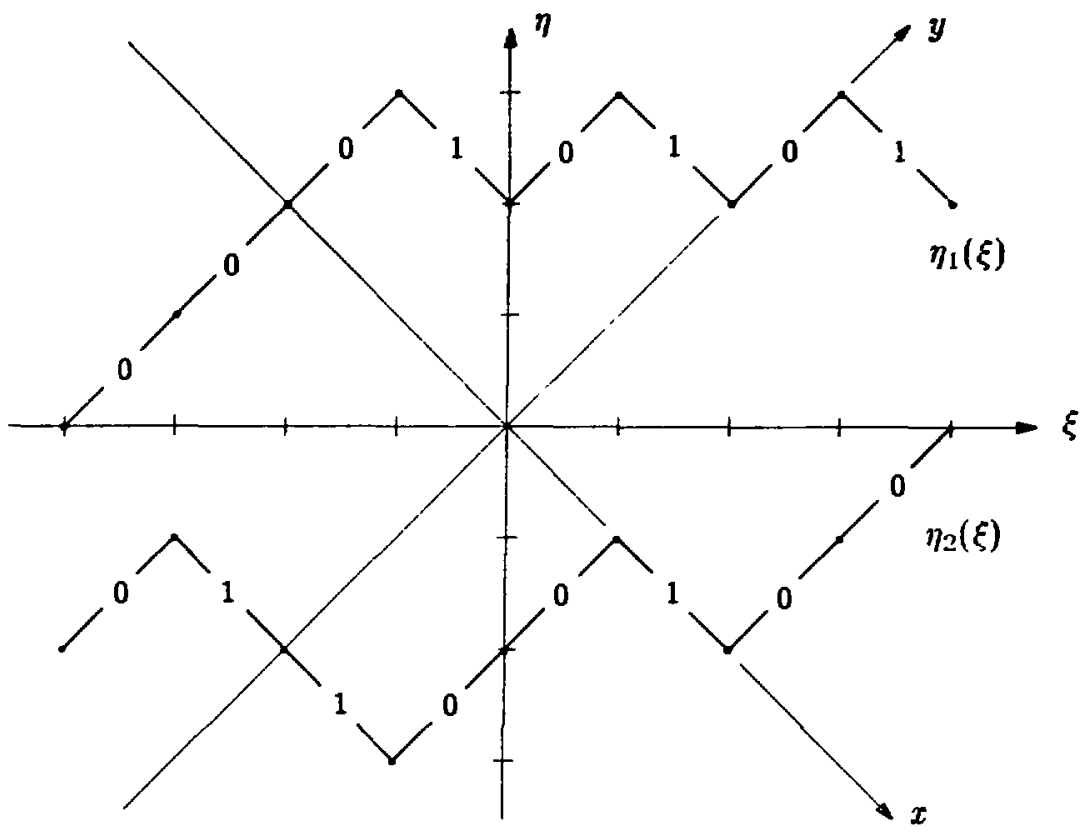

Figure 1

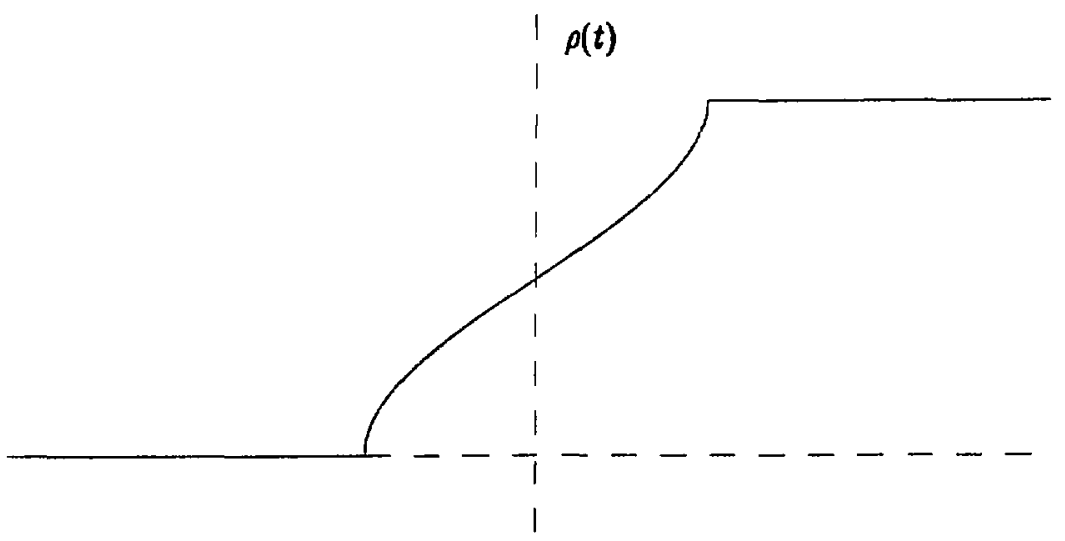

Figure 2 
THE LOOP GAS PICTURE OF THE LAMBDA TRANSITION 


\section{Introduction}

At present, the $\lambda$-transition in helium is by far the most accurately measured phase transition in nature. In an extremely difficult experiment that is in fact sensitive to the tiny pressure inhomogeneity due to the gravitational field, Lipa and $\mathrm{Chui}^{1}$ have come within $5 \times 10^{-8}$ $K$ of the $\lambda$-point. The major theoretical issue at stake is the value reported for the speciflc heat exponent:

$$
\alpha_{\mathrm{exp}}=-0.0127 \pm 0.0026
$$

The $\lambda$-transition is believed to belong to the family of critical phenomena characterized by a two-component order parameter. A renormalization group calculation with the $\phi^{4}$ fleld theory representative of such models yields the exponent ${ }^{2}$ :

$$
\alpha_{\text {theory }}=-0.007 \pm 0.006
$$

The agreement is in fact quite good since a priori there is not even a good reason why $\alpha$ should be so small.

The usual way of arriving at a two-component or $n=2$ model for the $\lambda$-transition is to identify the complex amplitude of the superfluid wave function as the order parameter ${ }^{3}$. This also points out a peculiarity unique to helium; namely, the physical impossibility of measuring the order parameter. To the uninitiated it must seem that the phase transition is somehow a conspiracy of the mathematics.

It is of course possible to give very sound physical arguments to supplement the remarks given above. The only problem is that there are many intervening steps and concepts that have to be deflned and elaborated upon. To the physicist it would be desireable to know directly what the atoms are doing. 
The microscopic basis of the $\lambda$-transition was first proposed by F. London ${ }^{4}$ and presented in more detail by Feynman ${ }^{5}$. While these eariy investigations were important in exposing the basic mechanism of the $\lambda$ transition, the relationship of these ideas to the modern, comprehensive theory of critical phenomena has not yet been made clear. The present work makes a modest effort in establishing this connection.

\section{Ideal Bose Condensation in Conflguration Space}

The fact that the partition function for the ideal Bose gas can be evaluated in configuration space has been discovered by several people ${ }^{8,7}$, including the author. The advantage of this method over the usual momentum space approach is that our insight about the nature of the condensation can easily be carried over to real helium.

For a system with $N$ identical bosons in a rolume $V$, we consider the symmetrized states

$$
|x\rangle_{B}=\frac{1}{\sqrt{N !}} \sum_{P}\left|x_{P_{1}} \cdots x_{P_{N}}\right\rangle
$$

where the sum is over all permutations

$$
P=\left(\begin{array}{ccc}
1 & \cdots & N \\
P_{1} & \cdots & P_{N}
\end{array}\right)
$$

and the particle positions have been denoted collectively by $x:=\left(x_{1}, \ldots, x_{N}\right)$. The partition function is then given by 


$$
\begin{aligned}
Z & =\operatorname{Tr} e^{-\rho H} \\
& =\frac{1}{N !} \int_{V} d^{3} x_{1} \cdots \int_{V} d_{N}^{3}\left\langle\left. x\right|_{\theta} e^{-\beta H} \mid x\right\rangle_{\sigma} \\
& =\frac{1}{N !} \sum_{P} z(P)
\end{aligned}
$$

where, using (2.1),

$$
z(P)=\int_{V} d^{3} x_{1} \cdots \int_{V} d^{3} x_{N}\left(x_{P_{1}} \cdots x_{P_{N}}\left|e^{-\beta H}\right| x_{1} \cdots x_{N}\right\rangle
$$

When $H$ is the hamiltonian for the ideal gas,

$$
H=\sum_{i=1}^{N} \frac{p_{i}^{2}}{2 m}
$$

the matrix element in (2.3) is just the diffusion kernel for $N$ noninteracting particles:

$$
z(P)=\int_{V} \frac{d^{3} x_{1}}{\lambda^{3}} \cdots \int_{V} \frac{d^{3} x_{N}}{\lambda^{3}} \prod_{i=1}^{N} \exp \left\{-\frac{m}{2 \beta}\left(x_{i}-x_{P_{i}}\right)^{2}\right\}
$$

where $\lambda$ is the thermal de Broglie wavelength

$$
\lambda=\frac{h}{\sqrt{2 \pi m k_{B} T}} .
$$

According to (2.2) the partition function is given in terms of a weighted sum over permutations. Since all the terms in the sum are positive, it is natural to ask which permutations give the greatest contribution. A permutation can be changed by relabeling the particles but this clearly will not affect the value of (2.3). We need therefore concentrate only on 
the character of the permutation that is unaffected by relabeling: the cycle structure. We implement this idea by writing

$$
P \in\left\{n_{1}, n_{2}, \ldots, n_{N}\right\}
$$

when $P$ contains $n_{1}$ 1-cycles, $n_{2} 2$-cycles, etc. . To compute the value of (2.3) it is now only necessary to know the numbers $n_{1}, n_{2}, \ldots$. These are in general any set of non-negative integers that satisfy the constraint

$$
N=\sum_{k=1}^{N} k n_{k}
$$

Let us now calculate (2.4) for permutations of the type (2.5). For each set of paricles that comprise a cycle, the integrations are independent of all the other particle positions. Consider the case of a cycle of $k$ particles, where $k$ is not too large $\left(y_{k}=y_{0}\right)$ :

$$
\int_{V} \frac{d^{3} y_{1}}{\lambda^{3}} \cdots \int_{V} \frac{d^{3} y_{k}}{\lambda^{3}} \prod_{i=1}^{k} \exp \left\{-\frac{m}{2 \beta}\left(y_{i}-y_{i-1}\right)^{2}\right\}=\frac{V}{\lambda^{3}} \frac{1}{k^{3 / 2}} .
$$

Geometrically, we are integrating over the configurations of a closed chain or loop, the links of which are separated by a distance of order $\lambda$. When $k \sim O(1)$ the size of the loop is much smaller than the volume $V$. It is then justifled to replace the integration over the loop's center of mass position by the factor $V$.

We will now proceed under the assumption that the important permutations are composed entirely of small cycles. We will see later that this assumption leads to sensible results for temperatures above a certain critical temperature. Below this temperature there are large loops where (2.7) no longer applies. For now, we observe that

$$
n_{1}, n_{2}, \ldots, n_{k}, \ldots \sim O(N)
$$


with relative magnitudes that are expected to decrease rapidly with the cycle length $k$.

Taking the product of the factors (2.7) for each of the cycles in $P$, we can write the partition function in the form

$$
Z_{I B G}=\sum_{\left\{n_{1}, n_{2}, \ldots\right\}} \frac{1}{N !} \mathcal{C}\left(n_{1}, n_{2}, \ldots\right) \prod_{k}\left(\frac{V}{\lambda^{3}} \frac{1}{k^{3 / 2}}\right)^{n_{k}}
$$

where the sum is subject to constraint (2.6) and the combinatorial factor $\mathcal{C}\left(n_{1}, n_{2}, \ldots\right)$ counts the number of permutations having the cycle decomposition (2.5):

$$
\mathcal{C}\left(n_{1}, n_{2}, \ldots\right)=\frac{N !}{1^{n_{1}} 2^{n_{2}} \cdots n_{1} ! n_{2} ! \cdots} .
$$

Rather than do the actual sum in (2.9) it is sufficient, considering (2.8), to find the particular set of values of the numbers $n_{i}$ at which the sum is strongly peaked. Implementing the constraint (2.6) with a Lagrange multiplier $\mu$, the maximum occurs when

$$
\frac{n_{k}}{N}=\frac{V}{\lambda^{3} N} \frac{e^{-\mu k}}{k^{5 / 2}} \quad(k=1,2, \ldots)
$$

provided $\mu>0$ satisfles the equation

$$
\sum_{k=1}^{\infty} \frac{e^{-\mu k}}{k^{3 / 2}}=\frac{N \lambda^{3}}{V}
$$

Extending the sum in (2.12) to inflnity is justifled if it turns out that $\mu \sim O(1)$. In that case both (2.11) and (2.12) have sensible thermodynamic limits. Upon taking $N \rightarrow \infty, V \rightarrow \infty$ with $N \cdot / V=\rho$ we see that $n_{k} \sim O(N)$ and decays exponentially with $k$. Moreover, if we consider (2.12) at high temperatures with $\lambda \rightarrow 0$ we see that $\mu$ 
becomes large. According to (2.11) we then have $n_{1} \approx N$ with the other cycle numbers negligible. Of course, the preponderance of 1-cycles at high temperatures agrees with our concept of the classical partition function where the amplitudes of nearby particles do not interfere very much.

As the temperature is lowered, the admixture of larger cycles is increased. Ultimately we will encounter a difficulty because the rightband-side of (2.12) can be made arbitrarily large whereas the left-handside cannot. Consequently, our equations do not give a sensible result for temperatures below a critical temperature given by

$$
\sum_{k=1}^{\infty} \frac{1}{k^{3 / 2}}=2.612 \ldots=\frac{\rho h^{3}}{\left(2 \pi m k_{B} T_{c}\right)^{3 / 2}}
$$

This problem arises because we have not allowed for the possibility of very large cycles. Therefore, let us suppose that $N_{0}$ particles belong to large or 'macroscopic' cycles and the remainder, $N^{\prime}=N-N_{0}$, belong to small or 'flnite' cycles. The terminology has been chosen to distinguish between those cycles that stay finite in the infinite volume limit and those that scale in size with the volume. For the ideal gas the two sets of particles do not interact and the partition function can be written as the product

$$
Z_{I B G}=Z_{0} Z^{\prime}
$$

Since the previous analysis for the case of finite cycles applies to $Z^{\prime}$, we can borrow our results with $N$ replaced b $y^{\prime} N^{\prime}$. In particular, we will be interested in the logarithm of (2.9) when the cycle numbers (2.11) have been substituted:

$$
\log Z^{\prime}=N^{\prime}\left\{\mu+\frac{V}{\lambda^{3} N^{\prime}} \sum_{k=1}^{\infty} \frac{e^{-\mu k}}{k^{5 / 2}}\right\},
$$


where $\mu$ is now determined by

$$
\sum_{k=1}^{\infty} \frac{e^{-\mu k}}{k^{3 / 2}}=\frac{N^{\prime} \lambda^{3}}{V}
$$

Earlier we argued that (2.14) ceases to make sense below a certain critical temperature. Alternatively, we could have concluded that there is an upper limit on the number of particles:

$$
N^{\prime} \leq N(T)=2.612 \frac{V}{h^{3}}\left(2 \pi m k_{B} T\right)^{3 / 2}
$$

For the strict inequality we have $\mu>0$ while at the upper limit $\mu=0$.

From equations (2.13) and (2.14) it is easy to derive the relation

$$
\frac{\partial}{\partial N^{\prime}} \log Z^{\prime}=\mu
$$

which shows that $\mu$ can be identifled with the (negative) chemical potentiai. As long as $\mu>0$, the value of $Z^{\prime}$ can be increased by adding more particles. A possible external source of particles is the set of $N_{0}$ particles in $Z_{0}$. Before we can decide whether it is advantageous to transfer particles from $Z_{0}$ to $Z^{\prime}$ we have to evaluate $Z_{0}$.

By definition, the cycles appearing in $Z_{0}$ are all macroscopic. Alternatively, we can say that the number of cycles in $Z_{0}$ is not macroscopic (i.e. much less than $O(N)$ ). Therefore, unless the contribution of each cycle in $Z_{0}$ goes like the exponential of $N$, the limit $N \rightarrow \infty$ of $N^{-1} \log Z_{0}$ will ranish.

To show that this is indeed the case one has to be more careful about evaluating (2.7). The first step is to realize that the contribution of a cycle is just the trace of a single particle kernel: 


$$
\begin{aligned}
\int_{V} d^{3} x_{1} \cdots \int_{V} d^{3} x_{k} & \left\{x_{1}\left|\exp \left(-\beta \frac{p^{2}}{2 m}\right)\right| x_{2}\right\rangle \cdots\left\langle x_{k}\left|\operatorname{exF}\left(-\beta \frac{p^{2}}{2 m}\right)\right| x_{1}\right\rangle \\
& =\int_{V} d^{3} x_{1}\left(x_{1}\left|\exp \left(-\beta k \frac{p^{2}}{2 m}\right)\right| x_{1}\right\rangle \\
& =\sum_{E_{i}} \exp \left\{-\beta k E_{i}\right\} .
\end{aligned}
$$

The flnal sum is over the single particle energy levels. Since the spacing of low lying energy levels is $O\left(V^{-2 / 3}\right)$ or $O\left(N^{-2 / 3}\right)$, we consider two cases. First, if $k \ll N^{2 / 3}$ the sum can be replaced by an integral and one obtains (2.7). If instead, $k>N^{2 / 3}$, it is sufficient to keep just the first term. Since the ground state energy is of the same order as the level spacings, we obtain the estimate

$$
k \text {-cycle } \sim O\left(\exp \left(-c k N^{-2 / 3}\right)\right) \quad\left(k>N^{2 / 3}\right)
$$

Thus we see that not even the longest cycles (with $k \sim O(N)$ ) enter as $O(\exp (-c N))$ factors in $Z_{0}$.

The lect that the number of cycles in $Z_{0}$ is much less than $N$, has lead to the conclusion that the free energy contributed by $Z_{0}$ is not extensive. In other words, the partirles in $Z_{0}$ have effectively zero perparticle free energy in the thermodynamic limit. This means that as long as $\mu>0$ one can transfer particles from $Z_{0}$ to $Z^{\prime}$ and thereby increase the value of the total partition function. The largest partition function is attained when the inequality (2.15) is saturated. Accordingly, the total free energy comes from a subset $N(T)$ of the particles while the remaining particles have the role of a 'condensate'.

We will now show how the basic pruperty of the condensation can be extended to an interacting Bose liquid such as helium. A useful starting point is equation (2.2) since it makes no reference to the detailed 
interactiuns among the particles. In principle, it is possible to evaluate the Schrödinger kernels $(2.3)$ that define $z(P)$ for various pernutations $P$. We can then regard (2.2) as an effective partition function for permutations. Guided by our experience with the ideal Bose gas, we use a particular property of this partition function to define a criterion for oondensation:

"Typical permutation

"Bose Condensation" $\Leftrightarrow$ contains a macroscopic cycle".

By 'typical' we mean that the relative probability of 'atypical' permutations go to zero in the thermodynamic limit. Similarly, a proper deflnition of 'macroscopic' also requires the thermodynamic limit.

The appearance of macroscopic cycles below the critical temperature in the Bose liquid can be compared with the appearance of magnetic domains below the Curie temperature in the ferromagnet. The singular onset of some macroscopic property is recognized as a general feature of critical phenomena and usually has immediate physical consequences that can be measured in the laboratory. Unfortunately, no experiment will ever be able to observe macroscopic cycles in superfluid helium. These cycles are, after all, only a mathematical artifice. We recall that the average over permutations was necessary only because initially we decided to give each particle a fictitious identity. The analog of the non-plysical nature of the 'order parameter' in the more familiar approach shows up in the impossibility of directly measuring the phase of the superiluid wavefunction.

\section{Of Diagonal L.ong Range Order}

In the Bast Section the Bose condensed phase was characterized by the appearance of macroscopic cycles in the effective partition function 
for permutations. Previous rigorous deflnitions of the condensatinn have relied on the notion of off diagonal long range order (ODLRO) ${ }^{8}$. By illustrating ODLPO in graphical terms we can show that the two ideas are in fact equivalent.

The basic mathematical object considered by 'ODLRO is the socalled 'reduced' density matrix:

$$
\rho\left(x, x^{\prime}\right)=\frac{1}{N !} \int_{V} d^{3} x_{1} \cdots \int_{V} d^{3} x_{N}\left(\left.x_{1} \cdots x_{N} x^{\prime}\right|_{B} e^{-\beta H} \mid x_{1} \cdots x_{N} x\right)_{B}
$$

The criterion for condensation is the statement:

$$
\lim _{\left|x \rightarrow x^{\prime}\right| \rightarrow \infty} \rho\left(x, x^{\prime}\right)=\text { const }>0
$$

This behavior is in contrast to that of the non-condensed phase where $\rho\left(x, x^{\prime}\right)$ decays exponentially as a function of the separation $\left|x-x^{\prime}\right|$.

The particle states in (3.1) are symmetrized and the matrix element can be written as a sum of terms involving permutations as in the previous section. The only difference is the presence of the positions $x$ and $x^{\prime}$ that are not shared between the two vectors. Thus, whereas the range of each permutation is the set of $N+1$ particle positions $\left\{x_{1}, \ldots, x_{N}, x\right\}$, the image of each permutation is the set $\left\{x_{1}, \ldots, x_{N}, x^{\prime}\right\}$. Graphically, each permutation can be represented by a collection of cycles and a chain. For every permutation $P$, the chain begins at $x$ and conitinues to $P(x)$, $P^{2}(x)$, etc. . This sequence must stop at $P^{k}(x)=x^{\prime}$ for some $k$, since $x^{\prime}$ is not in the range of $P$. The remaining positions all belong to cycles since each of them is an element of both the range and inage of $P$.

The reduced density matrix $\rho\left(x, x^{\prime}\right)$ measures the likelihood of having the points $x$ and $x^{\prime}$ connected by a chain. In the condensed phase, criterion (3.2) tells us this probability approaches a fixed, nonzero value as ihe points are separated by a large, in fact, macroscopic distance. 
This statement is not so very different from our earlier observation about macroscopic cycles. Namely, in the condensed phase there is a nonzero probability that $t$ wo widely separated particles, picked at random, belong to the same cycle.

\section{The Dilute Hard Sphere Gas}

The actual $\lambda$-transition differs in essential details from the condensation of the ideal gas. Even though the basic mechanism of ithe condensation can be discussed in general terms without reference to the interactions among the particles, it is nevertheless the case that the interactions contribute signiflcantly to the mathematical signature of the phase trans:tion. In order to appreciate the effects of interactions we will consider a dilute gas of hard spȟeres.

For an interparticle potential of the form

$$
V\left(x_{1}, x_{2}\right)=\left\{\begin{array}{cl}
0 & \left|x_{1}-x_{2}\right|>a \\
\infty & \left|x_{1}-x_{2}\right|<a
\end{array}\right.
$$

the quantum mechanical two-body problem can be solved exactly. The two-particle kernel is given by the expression

$$
\begin{aligned}
& \left.\left\langle x_{1}^{\prime} \tau_{2}^{\prime}\right\} \exp \left\{-\beta\left[\frac{p_{1}^{2}}{2 m}+\frac{p_{2}^{2}}{2 m}+V^{\prime}\left(x_{1}, x_{2}\right)\right]\right\} \mid x_{1} x_{2}\right) \\
& =\frac{1}{\lambda^{6}} \exp \left\{-\frac{m}{2 \beta}\left[\left(x_{1}-x_{1}^{\prime}\right)^{2}+\left(x_{2}-x_{2}^{\prime}\right)^{2}\right]\right\}\left[1-a F\left(y, y^{\prime}\right)+O\left(a^{2}\right)\right]
\end{aligned}
$$

where

$$
y=x_{2}-z_{1} \quad y^{\prime}=x_{2}^{\prime}-z_{2}^{\prime}
$$


are the relative coordinates and

$$
F\left(y, y^{\prime}\right)=\frac{|y|+\left|y^{\prime}\right|}{|y|\left|y^{\prime}\right|} \exp \left\{-\frac{m}{4 \beta}\left[\left(|y|+\left|y^{\prime}\right|^{2}-\left(y-y^{\prime}\right)^{2}\right]\right\} .\right.
$$

We expect Bose condensation to occur when the distance between nearby particles, $f^{-1 / 3}$, is comparable with the thermal wavelength $\lambda$. Using the fact that the gas is dilute, or $\rho a^{3}<1$, it follows that $a<\lambda$ near the $\lambda$-point. it was for this reason that we retained only the s-wave piece of (4.1).

We nov propose the following approximate partition function for the dilute gas of hard spheres:

$$
\begin{gathered}
Z_{D G}=\frac{1}{N !} \sum_{P} z(P) \\
z(P)=\int_{V} \frac{d^{3} x_{1}}{\lambda^{3}} \cdots \int_{V} \frac{d^{3} x_{N}}{\lambda^{3}} \prod_{i=1}^{N} \exp \left\{-\frac{m}{2 \beta}\left(x_{i}-x_{P_{i}}\right)^{2}\right\} \\
\prod_{i<j}\left\{1-a F\left(x_{i}-x_{j}, x_{P_{i}}-x_{P_{j}}\right)\right\} .
\end{gathered}
$$

This partition function correctly accounts for all possible pairs of twoparticle scatterings. The kinds of quantum mechanical effects that have not been included involve scattering processes that do not factor into the prcduct of two-particle scatterings. Such effects are expected to be small in the dilute gas limit.

As in the problem of the ideal gas, it is advantageous to decompose each permutation into a product of cycles. In the high temperature phase when there are no macroscopic cyc!es, the configurations resemble a gas of 'loops' in space. Each loop is a closed chain of particle positions connected by 'links' which interact weakly. Thus, in addition to interactions among different loops, an indiviuual loop may have interactions among its own links. 
It is possible to evaluate (4.2) approximately along the lines of a Mayer expansion. In analogy with the ideal gas, one assumes there are $n_{1}$ 1-cycles, $n_{2}$ 2-cycles, etc. and maximizes the free energy as a function of these numbers subject to the constraint that the total number of particles is unchanged. To lowest order, the interactions of the hard spheres will add contributions to the free energy proportional to $n_{i}$ and $n_{i} n_{j}$. These terms correspond to a single interaction between two links, either on the same loop or on two different loops.

Fortunately, the 'cluster integrals' required to compute the interactions can be evaluated exactly. We do not supply these details because, not surprisingly, the final answer for the free energy agrees exactly with an earlier calculation by Huang ${ }^{\theta}$ using a different method:

$$
\begin{aligned}
N^{-1} \log Z_{D G}-\mu & =\frac{1}{\rho \lambda^{3}}\left[f_{5 / 2}-2 \frac{a}{\lambda} j_{3 / 2}^{2}+O\left((a / \lambda)^{2}\right) .\right] \\
1 & =\frac{1}{\rho \lambda^{3}}\left[f_{3 / 2}-4 \frac{a}{\lambda} f_{1 / 2} f_{3 / 2}+O\left((a / \lambda)^{2}\right)\right]
\end{aligned}
$$

where

$$
f_{n / 2}=\sum_{k=1}^{\infty} \frac{e^{-\mu k}}{k^{n / 2}} .
$$

Equations (4.3) and (4.4) are the direct counterparts of equations (2.13) and (2.14) respectively, of the ideal gas (here we consider only $T>T_{c}$ where $N^{\prime}=N$ ).

Although we said earlier that the partition function (4.2) should accurately describe the dilute hard sphere gas near the phase transition, we will now argue that the expansion outlined above breaks down in that region. In the original partition function each link has some small probability of interacting with one of the nearby links. In other words, an equilibrium configuration will involve some probability of interaction 
per particle. The 'Mayer expansion' of (4.2), however, has assumed that the number of interactions per loop is small. The correction terms in (4.3) and (4.4) take into account only one interaction per loop, independent of the size of the loop. On the high temperature side of the phase transition the distribution of loop lengths is exponential,

$$
\frac{n_{k}}{N}=\frac{1}{\rho \lambda^{3}}\left[\frac{e^{-\mu k}}{k^{5 / 2}}+O(a / \lambda)\right],
$$

with a characteristic number of particles per loop given by $\mu^{-1}$. In the vicinity of the $\lambda$-point where this number diverges, any finite expansion in the number of interactions per loop is bound to fall seriously short of the actual number of interactions.

The kind of configuration where the 'loop' expansion would apply is shown schematically in figure 1 (interactions are represented by dots). Since the probability for any link to interact is of order $a / \lambda$ while the number of links per loop is of order $\mu^{-1}$, we require

$$
\left(\frac{a}{\lambda}\right) \frac{1}{\mu} \ll 1
$$

if the total number of interactions per loop is small. As long as the correction terms in (4.4) are small, the behavior of $\mu$ near the ideal gas critical temperature is given by

$$
\mu(t) \sim(\text { const }) t^{2}
$$

where $t=\left(T-T_{c}\right) / T_{c}$ is the reduced temperature. Consequently, if

$$
t \sim \sqrt{\frac{a}{\lambda}} \approx\left(a^{3} \rho\right)^{1 / \beta}
$$

then our expansion no longer makes much sense because the configurations are no longer 'loop-like' but appear as shown in figure 2 . 
The correct way of analyzing (4.2) in the vicimity of the critical point is suggested by figure 2. Here, a typical loop can experience a fair number of interactions with jtself as well as with other loops so that the overall pattern is quite complicated. Our mental image of the situation is considerably simplifled if instead of thinking in terms of loops we now focus on the interacting link-pairs. Since the interactions are very weak these pairs appear as a dilute gas and are interconnected by very long chains of non-interacting links.

Although short chains and small non-interacting loops are also present, we expect the phase transition to be mediated by the long chains and loops. A particular advantage of long chains and loops is that they allow two useful simplifications that will be used in the following discussion. First, it is adequate to express the number of links in such objectsin terms of continuous variables. Second, the cycle numbers $n_{k}$ for $k$ very large are essentially either 0 or 1 and do not lead to combinatorial complications.

A single interaction between two long chains can be represented by a vertex with four oriented edges as shown in figure 3 . The vertez defines four subchains having $t_{1}, \ldots, t_{4}$ links with arrows indicating the sense in which particles are permuted along the chains. For the moment, we will consider the endpoints of the chains to be fired at $x_{1}, \ldots, x_{4}$ and integrate over the positions of the other particles. Using the interaction $-a F\left(y, y^{\prime}\right)$ where $y$ and $y^{\prime}$ are as shown in flgure 3 , the integration can easily be carried out when the numbers $t_{1}, \ldots, t_{4}$ are large. The most convenient form of the answer leaves the integration over the center of mass position of the interacting links, $z$, still to be performed:

$$
-32 \pi^{2} \frac{a}{\lambda^{2}} \int d^{3} z \prod_{i=1}^{4} L\left(x_{i}-z, t_{i}\right)
$$

where 


$$
L(x-z, t)=\frac{1}{\lambda^{2} \sqrt{4 \pi t^{3}}} \exp \left\{-\frac{m}{2 \beta} \frac{(x-z)^{2}}{t}\right\} .
$$

Although we have succeeded in isolating the effect of an interactior between two long chains, we do not propose to evaluate the partition function by writing down all possible 'graphs', i.e. ways of coupling together terms of the form (4.5). Rather, we will show that these graphs are effectively generated in the perturbative expansion of a particular $\phi^{4}$ fleld theory.

We begin by 'amputating' the 'legs' of the vertex (4.5). Combining two such legs gives us a close relative of the propagator $\left(t=t_{1}+\right.$ $\left.t_{2}\right)$ :

$$
\begin{aligned}
\Delta\left(z-z^{\prime}, t\right) & =\int d^{3} x L\left(x-z, t_{1}\right) L\left(x-z^{\prime}, t_{2}\right) \\
& =\frac{1}{4 \pi \lambda t^{3 / 2}} \exp \left\{-\frac{m}{2 \beta} \frac{\left(z-z^{\prime}\right)^{2}}{t}\right\} .
\end{aligned}
$$

(Our way of distributing constant factors between the legs and the vertex is chosen for later convenience.) In the final form we would like to have only integrations over the vertex positions remaining. Therefore, one should sum (4.6) over all values of $t$, the number of particles in the chain. Since the total number of particles at our disposal is fixed, we have to include a chemical potential $\mu$ in the summation. The resulting propagator has the familiar form when written in momentum space:

$$
\begin{array}{rlrl}
\Delta(q) & =\int d^{3} z e^{-i q z} \int_{0}^{\infty} d t e^{-\mu t} \Delta(z, t) \\
& =\frac{1}{q^{2}+M^{2}} & \left(M^{2}=\frac{4 \pi \mu}{\lambda^{2}}\right) .
\end{array}
$$

It is also possible to have very large loops that do not interact at all. The contribution of such a loop of $k$ particles is given by equation (2.7) 
which we must remember to divide by $k$ to avoid overccunting (earlier, the same factor occured as $k^{n_{k}}$ in (2.10)). By including the chemical potential $\mu$, we can sum over all conflgurations of any number of loops of all sizes:

$$
\begin{gathered}
1+\frac{V}{\lambda^{3}} \int \frac{d t}{t} \frac{e^{-\mu t}}{t^{3 / 2}}+\frac{1}{2 !}\left(\frac{V}{\lambda^{3}} \int \frac{d t}{t} \frac{e^{-\mu t}}{t^{3 / 2}}\right)^{2}+\cdots \\
=\exp \left\{\frac{V}{\lambda^{3}} \int \frac{d t}{t} \frac{e^{-\mu t}}{t^{3 / 2}}\right\}
\end{gathered}
$$

We now proceed to rewrite this in a more suggestive form:

$$
\frac{V}{\lambda^{3}} \int \frac{d t}{t} \frac{e^{-\mu t}}{t^{3 / 2}}=\int \frac{V d^{3} q}{(2 \pi)^{3}} \int_{O(1)}^{\infty} \frac{d t}{t} \exp \left\{-t\left(\frac{\beta}{2 m} q^{2}+\mu\right)\right\}
$$

The lower limit of the $t$ integral is $O(1)$ since a loop must contain at least one particle. However, this limit is irrelevant since we are really interested in the divergence at the $t \rightarrow \infty$ limit when $q \rightarrow 0$ and $\mu \rightarrow 0$ :

$$
\int_{O(1)}^{\infty} \frac{d t}{t} \exp \left\{-t\left(\frac{\beta}{2 m} q^{2}+\mu\right)\right\} \sim-\log \left(q^{2}+M^{2}\right) .
$$

Substituting into (4.8) gives the desired formal result:

$$
\begin{aligned}
\exp \left\{\frac{V}{\lambda^{3}} \int \frac{d t}{t} \frac{e^{-\mu t}}{t^{3 / 2}}\right\} & =\exp \left\{-\int \frac{V d^{3} q}{(2 \pi)^{3}} \log \left(q^{2}+M^{2}\right)\right\} \\
& =\left[\operatorname{det}\left(-\theta^{2}+M^{2}\right)\right]^{-1}
\end{aligned}
$$

There still remains the problem of taking into account the orientations, i.e. the directions in which the particles are permuted in the graph. It will be convenient to assign the labels 1 and 2 to the endpoints of each edge in such a way that the arrow associated with the edge points 
from 1 to 2 . If we define the matrix

$$
\left(e^{a b}\right)=\left(\begin{array}{ll}
0 & 1 \\
1 & 0
\end{array}\right)
$$

then the propagator from position/label $(x, a)$ to $(y, b)$ is given by

$$
\Delta^{a b}(x-y)=e^{a b} \Delta(x-y)
$$

where $\Delta(x-y)$ is the fourier transform of (4.7).

At each vertex an endpoint labeled 1 will be coupled with an endpoint labeled 2. This rule can be expressed in the usual way involving functional derivatives with respect to a two-component current $J_{a}(x)$ :

$$
v(x)=\frac{1}{8}\left(e^{a b} \frac{\delta}{\delta J_{a}(x)} \frac{\delta}{\delta J_{b}(x)}\right)^{2}
$$

where it is implied that $v(x)$ acts on the generating functional

$$
G[J]=\exp \left\{\frac{1}{2} \int d^{3} x \int d^{3} y J_{a}(x) \Delta^{a b}(x-y) J_{b}(y)\right\} .
$$

We now have all the necessary ingredients to write the expression for the general graph having any number of interactions and any number of non-interacting loops. The sum of all these graphs constitute the piece of the dilute gas partition function that we believe is relevant to the phase transition:

$$
Z_{D G}^{*}=\left.\sum_{n=0}^{\infty} \frac{(-g)^{n}}{n !} \int_{V} d^{3} z_{1} \cdots \int_{V} d^{3} z_{n} v\left(z_{1}\right) \cdots v\left(z_{n}\right) Z_{0}[J]\right|_{J=0}
$$

where

$$
g=32 \pi^{2} \frac{a}{\lambda^{2}}
$$


and

$$
Z_{0}[J]=\left[\operatorname{det}\left(-\partial^{2}+M^{2}\right)\right]^{-1} G[J]
$$

The change of variables

$$
\begin{aligned}
& J_{1}^{\prime}=\frac{1}{\sqrt{2}}\left(J_{1}+J_{2}\right) \\
& J_{2}^{\prime}=\frac{-i}{\sqrt{2}}\left(J_{1}-J_{2}\right)
\end{aligned}
$$

has the effect of simplifying (4.9) by replacing $e^{a b}$ everywhere it appears with $\delta^{a b}$. In terms of the transformed current, $Z_{0}\left[J^{\prime}\right]$ can ibe written as the functional integral

$$
Z_{0}\left[J^{\prime}\right]=\int[d \phi] \exp \left\{-S_{0}+\int d^{3} x J^{\prime} \cdot \phi\right\}
$$

where

$$
S_{0}=\int d^{3} x\left[\frac{1}{2}\left(\partial^{i} \phi\right) \cdot\left(\partial_{i} \phi\right)+\frac{1}{2} M^{2} \phi \cdot \phi\right]
$$

and $\phi$ is a two-component field. (We note that the determinant in (4.10) is in fact the square of the Gaussian-integration factor for single component flelds.) The functional derivatives can now be carried out and we recognize (4.9) as just the order by order expansion of a fleld theory with quartic interaction:

$$
\begin{gathered}
Z_{D G}^{*}=\int[d \phi] \exp \left\{-S_{D G}\right\} \\
S_{D G}=S_{0}+\int_{V} d^{3} x \frac{1}{8} g(\phi \cdot \phi)^{2} .
\end{gathered}
$$

Supplemented with the definition of $\mu$ in terms of the number of particles,

$$
-\frac{\partial}{\partial \mu} \log Z_{D G}^{*}=N
$$


our translation of the dilute gas partition function into a fleld theoretic model is now complete.

The two-component $\phi^{4}$ fleld theory is believed to be the correct model of critical behavior even for real (strongly interacting) helium. Our derivation applies in the dilute gas limit where we have shown it is possible to obtain concrete expressions for the parameters in the action. Implicit also in the definition of a field theory is the spatial cut-off which in our case can be directis traced back to the thermal wavelength $\lambda$. We emphasize, however, that our main interest has been the method of derivation which is considerably different from the one usually given in the literature ${ }^{3}$. One particularly nice feature of our approach is that it points out the similarities and differences of the condensation in the ideal gas.

\section{The Origin of $n=2$}

In the last section we have seen yet another way of deriving the two-component or $n=2$ fleld theory model of the $\lambda$-transition. The diversity of the methods used in deriving this field theory point out the fact that there are many, essentially correct ways of thinking about liquid helium. We will try to exhibit this diversity by tracing ithe various origins of $n=2$.

The most direct derivation observes that the phase of the condensate wavefunction is the order parameter of superfluidity ${ }^{3}$. Since the phase is a planar spin, the critical behavior belongs to the $n=2$ category.

The lattice gas approach of Matsubara and Matsuda ${ }^{10}$ is slightly more mysterious. One first argues that due to the hard core interactions, the states of liquid helium can be adequately represented by the states 
of the lattice gas having (at most) single occupancy at each site. By associating the 0 or 1 value of particle occupation with the up or down state of a spin, it is easy to verify that corresponding matrix elements of $a_{i}^{\dagger} a_{j}+a_{j}^{\dagger} a_{i}$ and the spin- $\frac{1}{2}$ operator $\sigma_{x}(i) \sigma_{x}(j)+\sigma_{y}(i) \sigma_{y}(j)$ agree. The former is the kinetic energy operator of the lattice gas while the latter gives the interaction of the spin- $\frac{1}{2} x y$ model. The quantum nature of the spin is nct expected to influence the critical behavior so that once again we have planar spins or $n=2$.

In the loop gas approach, the details of which have been given in Section 4, the $n=2$ is reflected in the two possible ways of beginning a game of musical chairs. We have seen that the partition ifunction sum involves all possible arrangements of permutation cycles in space. In addition to the specification of the atomic positions, however, it is important also to keep track of the sense in which the atoms are permuting. It is this 'extra' multiplicity that is responsible for the $n=2$ of the field theory.

\section{A Lattice Model of the Loop Gas}

In Section 4 we considered the effect of interactions among the helium atoms in the dilute gas limit and obtained a fleld theory model that is believed to give the observed critical behavior. A different way of introducing interactions was first proposed by Kikuchi ${ }^{11}$, based on the earlier work of Feynman ${ }^{5}$. The Feynman-Kikuchi (FK) model is deflned on a regular lattice and is motivated by considerations of the equilibrium distribution of helium atoms.

In calculating the partition function (Schrödinger kernels, etc.) it is not actually necessary to average over the positions of all the atoms. It is suffient to pick just one arrangement of atoms that is representative 
of an equilibrium configuration. Of course, the equilibrium conflgurations can only be known once $t_{L}$ - kerneis are computed and the pattern of permutation cycles is favorable. However, a much more naive consideration suggests the equilibrium distribution of atoms in helium is very different from that of the ideal gas. The hard sphere character of the helium atoms first of ail requires that the atoms never come within a hard sphere diameter's distance of each other. Moreover, if we imagine each helium atom enclosed in a 'cell' formed by its hard sphere neighbors, then the properties of the ground state wave function of the enclosed atom lends additional weight to configurations where atoms are well separated. This last argument applies of course only when the thermal wavelength of the atom is at least as large as the interparticle distance (which is the case at the $\lambda$-point).

The elegant idea of the FK model is then to choose one conflguration where the atoms are well separated. For convenience one chooses the complete set of sites of a simple cubic lattice. It should become evident later that the regularity of the arrangement of atoms will not introduce spurious effects.

Having already made one approximation, we proceed to make two more. Since we are only interested in the range of temperatures where the thermal wavelength is roughly one lattice unit, it is unlikely that atoms are permuted between distant sites. We therefore impose the restriction on the allowed permutations that every atom be permuted either to its original site or to one of the six nearest neighbor sites. The flinal approximation concerns the evaluation of the Schrödinger kernels. When the atoms are well separated, the permutation of a given atom should not be too much affected by what the other atoms are doing. It is therefore only necessary to know the relative values of the kernels for permuting zero or one lattice unit. If these were free kernels the ratio Foulù be 


$$
\frac{\text { one lattice unit }}{\text { zero lattice units }}=\exp \left\{-\frac{m a^{2}}{2 \beta}\right\}=e^{-t}
$$

where $a$ is the lattice spacing. Although it is not correct to use the free kernels, the ratio above (however we compute it) just deflnes a parameter in our model. The behavior with respect to changing the rescaled temperature $t$ is expected to be correct qualitatively since for large $t$ when it is difflcult for atoms to permute very far, the ratio is small.

Wi:h the three approximations considered, the evaluc ion of the helium partition function is reduced to a statistical mechanics problem involving permutation cycles on a cubic lattice. Geometrically, a conflguration can be visualized as a set of closed paths on the edges of the lattice such that every site is visited by at most one path (and o ce only). The set of sites that are not visited by a closed path represent all the atoms in 1-cycles. The weight of a configuration is the product of 'se kernels for all the particles. Since the particles in nontrivial $c_{j}$ cles co in ibute the faotor $e^{-t}$ relative to the particles in 1-cycles, the total weigh, is just $e^{-t L}$ where $L$ is the total length of path. Of course, in summing over conflgurations of paths one should count the two possible orientations of each (nontrivial) sycle separately.

The 2-cycle is a special case because it ir rolves a path that backtracks on itself. All the larger cycles are of the usual non-backtracking, self-avoiding variety. Kikuchi analyzes his model using mean field techniques and is able to incorporate the 2-cycle in the calculation. In the present work we choose to throw out the 2-c/cle because it complicates a Monte Carlo algorithm. Whether the 2-cyc'a is thrown out or not should have little effect on the critical behavior $\nabla$ hich we believe is due to the large cycles.

An interesting comparison with an entirely different lattice modei of the $\lambda$-transition can be made if the $F K$ wodel is expressed in terms of explicit edge or link variables $t_{i}$. By letting each link take on the walues 
0 or \pm 1 , we can represent both the presence of a path and its orientation (using the sign). To this we must add the constraint that (1) there is zero 'link flux' into any site and (2) no more than two active $\left(l_{i}= \pm 1\right)$ links impinge on any site. The FK partition function can now be written in the form

$$
Z_{F K}=\sum_{\substack{l_{i}=0, \pm 1 \\ \text { constraints: (1), (2) }}} \exp \left\{-t \sum_{i}\left|l_{i}\right|\right\}
$$

This bears a strong resemblance to the duality transformed $x y$ model studied recently by Dasgupta and Halperin ${ }^{12}$ :

$$
Z_{d u a l x y}=\sum_{\substack{l_{i}=\text { integer } \\ \text { constraint (1) }}} \exp \left\{-t \sum_{i} f_{i}^{2}\right\} .
$$

The restriction to integers with absolute value bounded by one in (6.1) is qualitatively compensated for by the use of the square in the exponentiai of (6.2). The configurations of the dual $x y$ model may also be thought of in terms of a collectica of closed paths but wir. the difference that patbs may overlap causing the absolute value of some links t's be greater than one. In taking the square, the 'energies' of pains do not add linearly creating an effective repulsion that favors paths which do not overlap. The important configurations are therefore very similar to those of the FK model.

In the original paper on the FK model ${ }^{11}$, Kikuchi obtains clear evidence of a phase transition using mean fleld methods. Unfortunately, the specifie heat develops a finite discentinuity in contrast to the nearly logarithmic singularity observed in real helium. We nould like to now show by using high-temperature expansion and Monte Carlo techniques that the phase transition appears to be of the correct type. in addition, me would like to peint out the role of large loops by fresenting evidence of their sudden appearance just below the critical temperature. 
At high temperatures the important conflgurations appear as a dilute gas of small loops (4-, 6-, 8-cycles, etc. in the FK model). If the various kinds of small loops are interpreted as molecular species, then the relevant approximation method is the Mayer expansion for a non-ideal, multicomponent gas. Because no two loops in the gas are permitted to share a site in the lattice, the cluster integrals involve counting the number of ways of placing various kinds of loops in space so that this condition j violated. For a particular order in the calculation, one has to evaluate all the cluster integrals wherc the combined length of all the participating loops is a certain value. As an example, the work required ito obtain the term of order sixteen ranges fr $m$ the counting of all the self-avoiding closed paths of length sixteen to the equally tricky task of counting all the ways of arranging a connected set of four 4-cycles. By implementing and carefully checking the required counting algorithms on a computer, it has been possible to carry out the expansion to order eighteen.

Since only the even terms are nonzero, our expansion for the free energy per particle has relatively few terms $\left(z=e^{-t}\right)$ :

$$
\begin{aligned}
N^{-1} \log Z_{F K}= & 6 z^{4}+44 z^{6}+216 z^{8}+744 z^{10}+4484 z^{12} \\
& +93984 z^{14}+15795 . \quad \because \because 216323543_{3}^{18} z^{18} \\
& +\cdots
\end{aligned}
$$

Unfortunately, the series for the speciflc heat exhibits large oscillations and various Padé extrapolations indicate nearby singularities in the complex $z$-plane. Such slow convergence to asymptotic behavior is a not uneommon phenomenon in the loose-packed lattices ${ }^{13}$ (such as the simple cubic). Rather than extending the series to higher order, it is perhaps more worthwhile to develop the loop gas expansion on a close-packed lattice. Nerertheless, for a range of extrapclation techniques we find a singularity on the positive real axis that is within $3 \%$ of the critical point determined 
by Monte Carlo simulation. Although positive, the -peciflc heat exponent we obtain is small and ranges from 0.05 to 0.1 .

Figures 4 through 6 show typical ligurations of locps at the temperatures $t=1.3,1.4$, and 1.6 in a $F^{*}$. mociel with $10^{3}$ sites. These were generated by Monte Carlo simp' , เion using the Metropolis algorithm. The algorithm changes conflg: wons by trying to introduce an elementary 4-cycle (of the two possibre orientations) at all possible plaquettes in a sweep of the lattice. Five distinct kinds of changes are possible: (1) a 4-cycle can be created, (2) a 4-cycle can be annihilated (by adding to it the oppositely oriented one), (3) an already existing cycle can be modified, (4) two cycles can be merged, and (5) a single cycle can be split into two. of course, any change must also be consistent with the constraint that the paths are self-avoiding.

When the density of active links is suffliently large, just a few of the local update processes described above are capable of generating very large loops by joining together smaller ones. It is therefore not inconceivable that below some critical temperature $t_{c}$ a certain fraction of the lattice sites will be visited by macroscopic loops. As discussed in Section 2, this fraction should have a thermodynamic limit and is called the 'condensate'. Although the notion of a macroscopic loop is only well defined in the limit of an infinite volume, it is still possible to obtain accurate estimates of the condensate fraction $f_{0}$ from Monte Carlo simulations or a finite lattice.

We have used two different methods of estimating the ccndensate fraction on a $20^{3}$ lattice. Both methods assume that for $t<t_{c}$ the inflnite volume limit of the density cf flnite loops varies with their length according to

$$
\rho_{k} \sim \text { (const) } \frac{1}{k^{\delta}} \quad(k \rightarrow \infty) .
$$

(Recall that for the ideal gas $\delta=\frac{5}{2}$.) The two methods differ in the 
kinds of quantities that are measured. The flrst method relies on the total number of active links, $N^{*}$, which includes 'finite' as well as 'macroscopic' cycles. The contribution due to finite cycles is found by directly measuning the cycle numbers $n_{k}$ for $k=4,6,8, \ldots$ and extrapolating to the form (6.3) for large $k$. If we let $N$ be the number of lattice sites, then the first estimate is given by

$$
f_{0}=N^{-1}\left(N^{*}-\sum_{k=4}^{K} k n_{k}-\sum_{k=K+2}^{\infty} k N \rho_{k}\right) .
$$

At the temperatures studied it was found that in going from $K=\mathbf{4 0}$ to $K=50$ the value of $f_{0}$ changed by less than $1.5 \%$.

In the second method, the average lengths of the first, second, $\ldots r^{\text {th }}$-longest loops, or $\bar{L}_{r}$ were obtained. If macroscopic loops were absent and the distribution of large loops in our flnite lattice was given by (6.3), then it is easy to show that

$$
\bar{L}_{\tau}=(\text { const }) \frac{\Gamma\left(r-(\delta-1)^{-1}\right)}{\Gamma(r)} \quad(r=1,2,3, \ldots .)
$$

In trying to match Monte Carlo measurements to this form it soon becomes clear that a much better fit is achieved (especially for larger $r$ ) with the replacement $\bar{L}_{r} \rightarrow \bar{L}_{r+\theta}$ where $s \sim 4$ and varies slightly with temperature. We interpret this to mean that the first $s$ or so longest loops are macroscopic and the population of large finite loops really only begins with loops smaller than these. The second estimate of the condensate fraction is thus

$$
f_{0}=N^{-1} \sum_{r=1}^{s} \bar{L}_{r}
$$

It was found that a change of \pm 1 in $s$ produced a change of less than $5 \%$ in $f_{0}$. 
The two estimation methods were found to agree within the uncertainty of the second method for the range of temperatures shown plotted in figure 7 . The point with $f_{0}=0$ is not a true measurement but has been included to show the location of the peak in the speciflc heat curve at $t_{c} \approx 1.44$. The other data points each involved some $2.5 \times 10^{4}$ sweeps through the lattice.

In contrast to the condensate measurements, the 'measurement of the speciflc heat is relatively straightforward, being just the fluctuation in the number of active links:

$$
C=N^{-1}\left(\overline{\left(N^{*}\right)^{2}}-\left(\overline{N^{*}}\right)^{2}\right) .
$$

The Monte Carlo data obtained on a $20^{3}$ lattice with $6.4 \times 10^{4}$ sweeps per data point are shown plotted in figure 8.

A detailed analysis of the Monte Carlo data in tenms of critical exponents, etc. has not been attempted. Instead, we submit the qualitative behavior of the data as strong evidence that the FK model has a phase transition of the 'critical' type. From here one might proceed, for example, with a high statistics flnite-size scaling study to confirm the logarithmic character of the speciflc heat singularity.

The most important lesson to be learned from Monte Carlo simulations of the FK model is that analogous simulations of real helium might not be very different. A more correct model would have to evaluate the Schrödinger kernels in the presence of complicated interatomic potentials. In principle we know how to calculate these things; in practice it is certain to be very time consuming. However, these complications have nothing to do with the large number of atoms. Perhaps a 'first principles' calcuiation with only a few atoms can be carried out and used to improve the original FK model. In addition to giving the critical behavior at the $\lambda$-point, such a model would then also have something to say about the actual value of $t_{c}$. 


\section{Reference⿰}

(1) J. A. Lipa and T. C. P. Chui Phys. Rev. Lett. 512291 (1983)

(2) J. C. LeGuillou and J. Zinn-Justin Phys. Rev. B21 3976 (1980)

(3) V. L. Ginzburg and L. P. Pitaerskii Zh. Eksperim i Teor. Fiz. 34 1240 (1958); english translation: Soviet Phys. : JETP 34858 (1958)

(4) F. London Phys. Rev. 54947 (1938)

(5) R. P. Feynman Phys. Rev. 911291 (1953)

(6) T. Matsubara Prog. Theor. Phys. 6714 (1951)

(7) R. P. Feynman "Statistical Mechanics" p. 62 W. A. Benjamin Inc., Reading Mass. 1972

(8) C. N. Yang Rev. Mod. Phys. 34694 (1962)

(9) K. Huang in "Studies in Statistical Mechanics" Vol. 2, p. 3 (eds. J. de Boer and G. E. Uhlenbeck) North-Holland Pub. Co., Amsterdam 1964

(10) T. Matsubara and H. Matsuda Prog. Theor. Phys. 16569 (1956)

(11) R. Kikuchi Phys. Rev. 96563 (1954)

(12) C. Dasgupta and B. I. Halperin Phys. Rev. Lett. 171556 (1981')

(13) see, e.g., D. S. Gaunt and A. J. Guttmann in "Phase Transitions and Critical Phenomena" Vol. 3, p. 181 (eds. C. Domb and M. S. Green) Academic Press, London 1974 
Some additional references on the subject of 'loops' are:

(14) H. K. Moffatt J. Fluid Mech. 35117 (1969)

(15) S. F. Edwards Proc. Phys. Soc. 91513 (1967)

(16) S. F. Edwards J. Phys. A 115 (1968) 


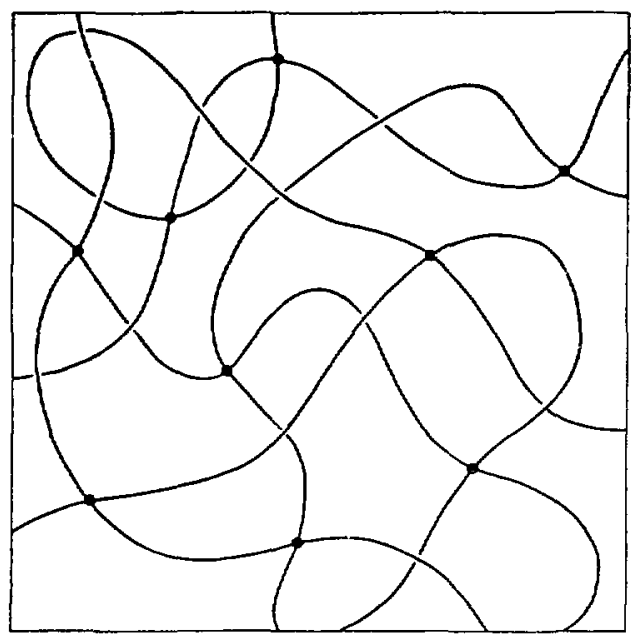

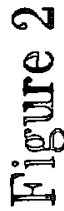

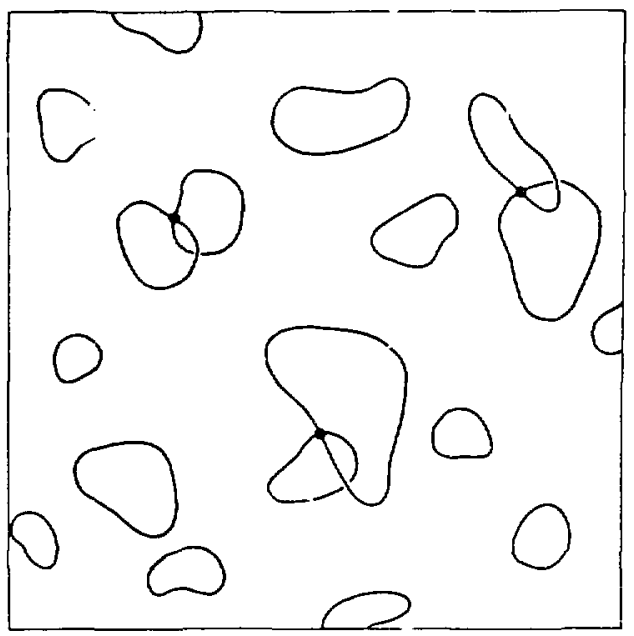

5 

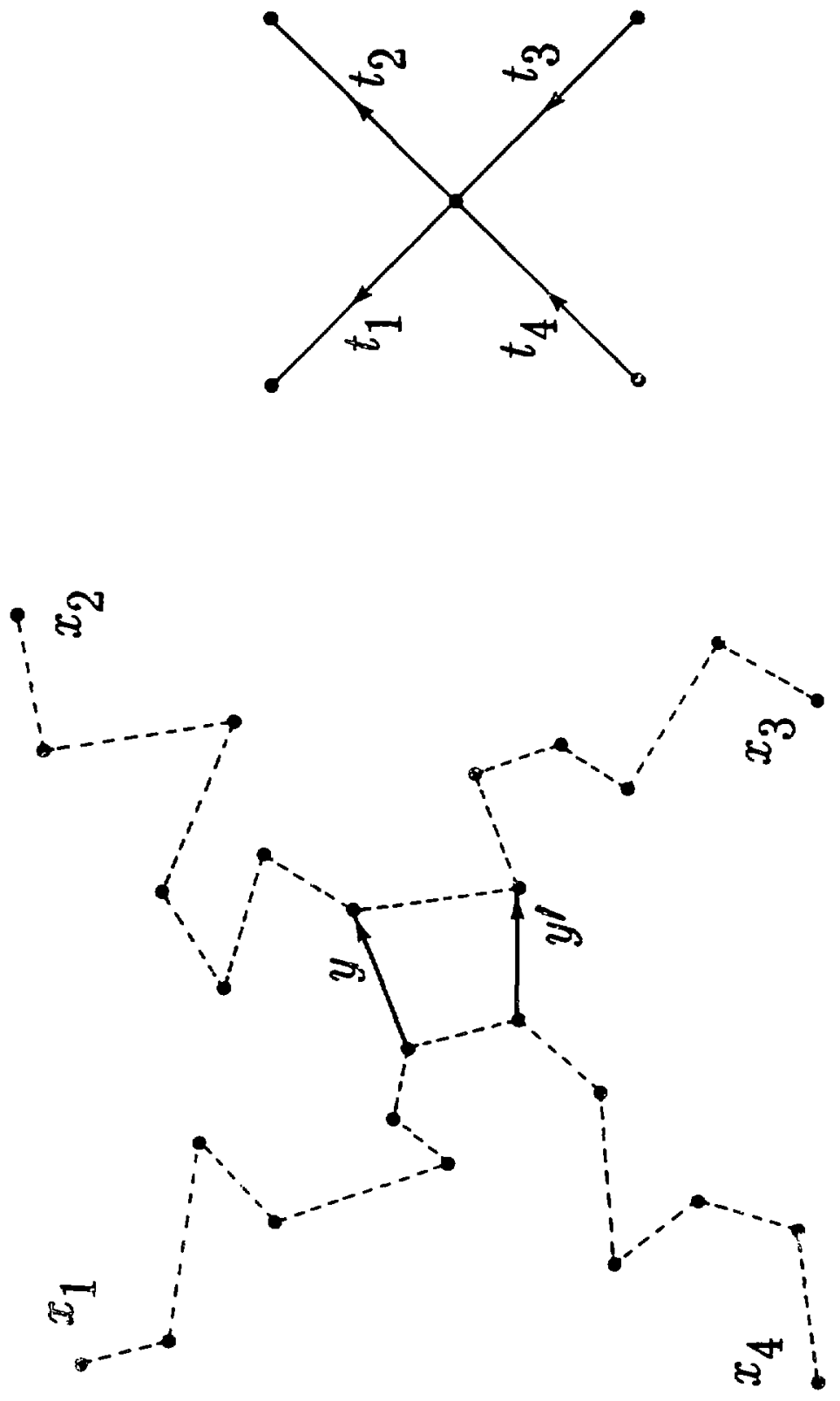

$\infty$ 


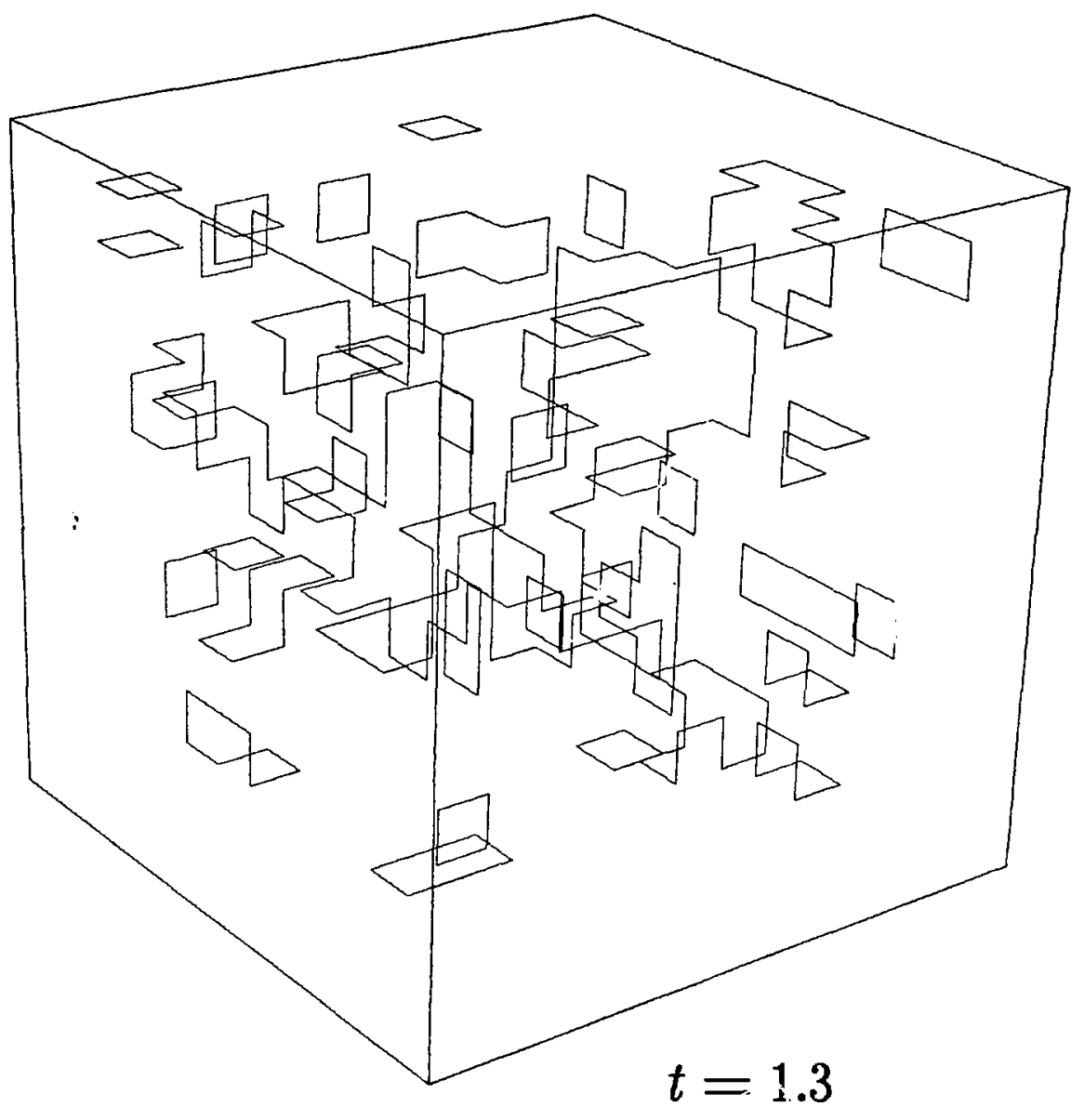

Figure 4 


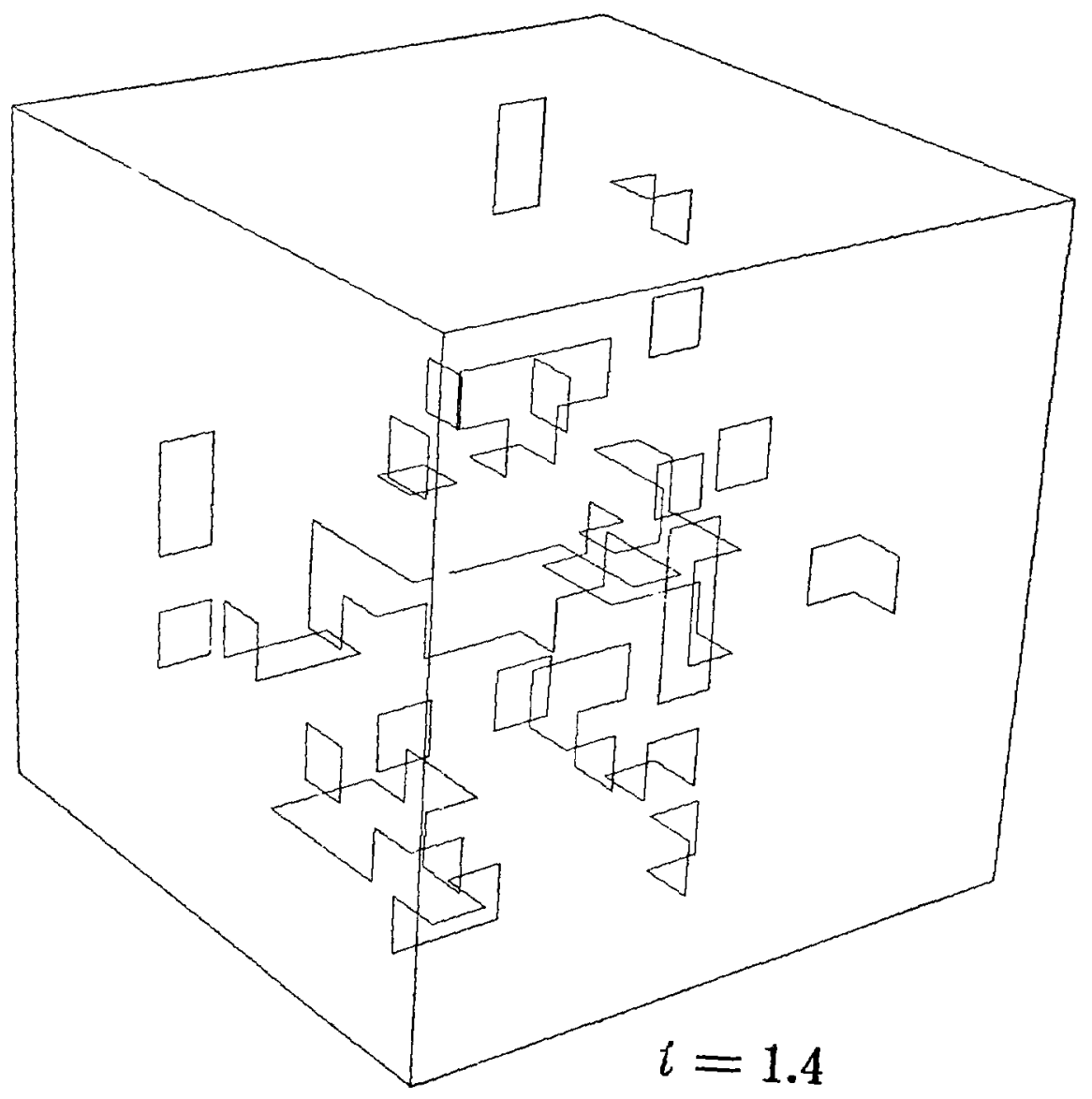

Figure 5 


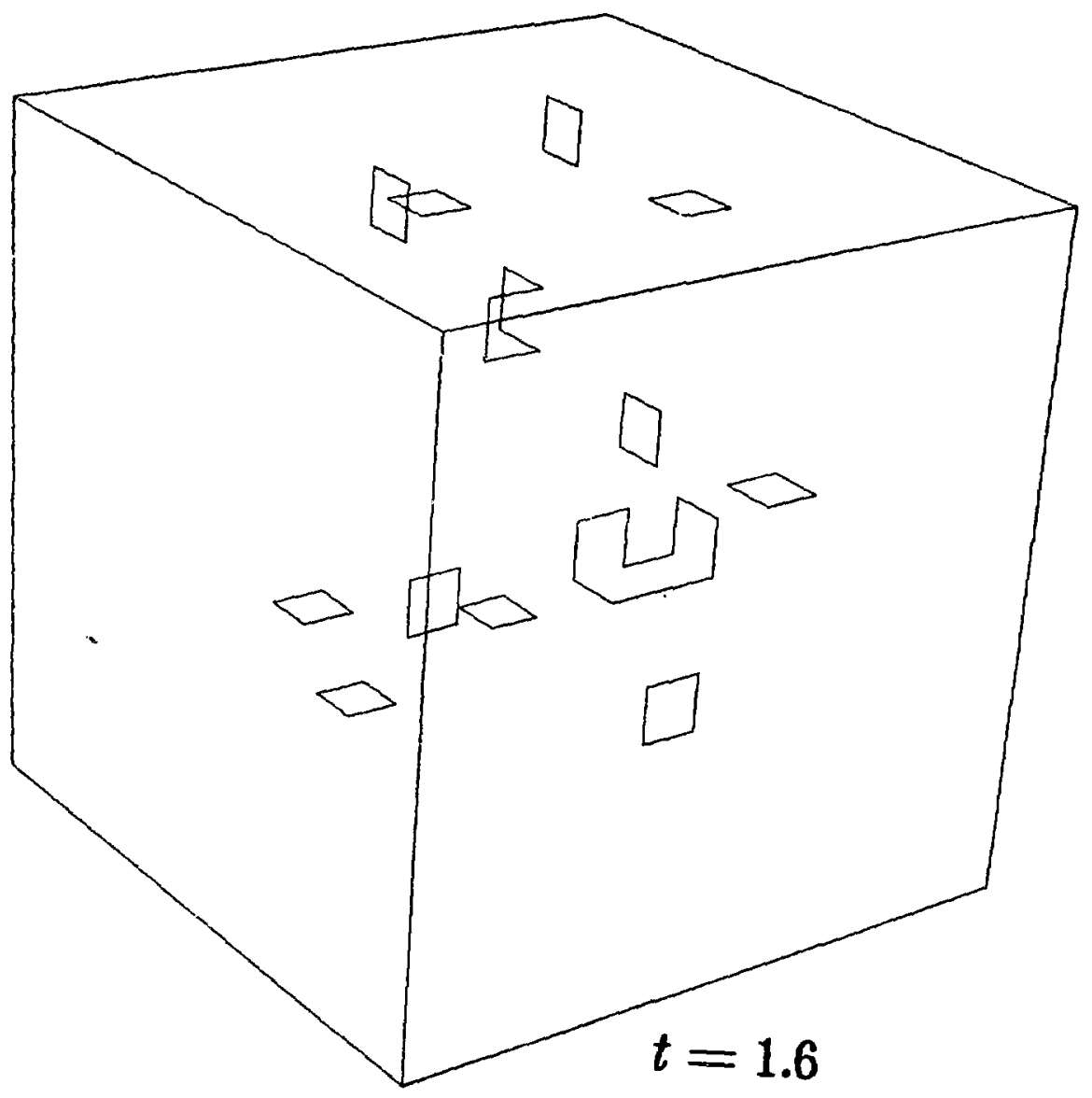

Figure 6 


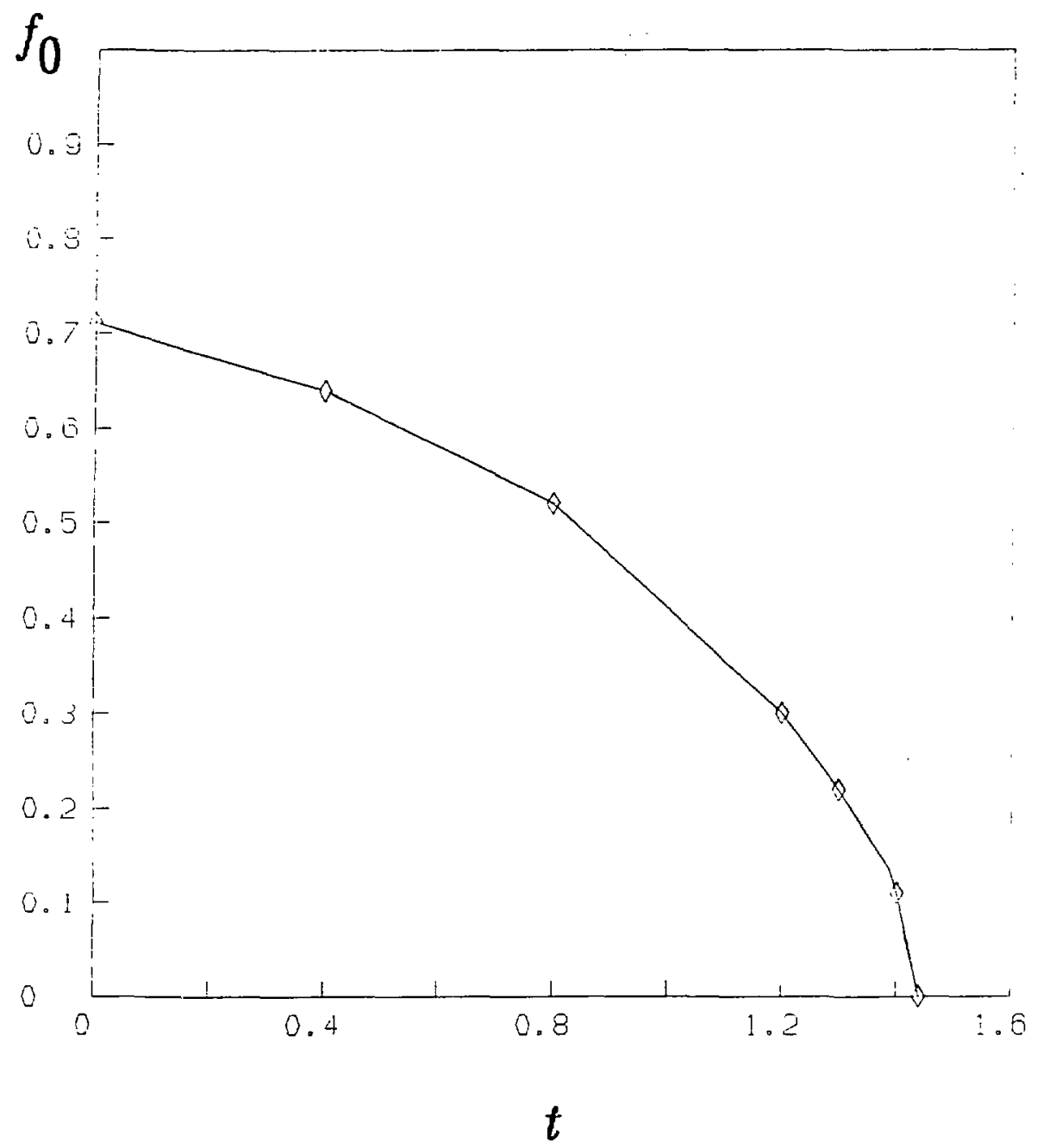

Figure 7 
86

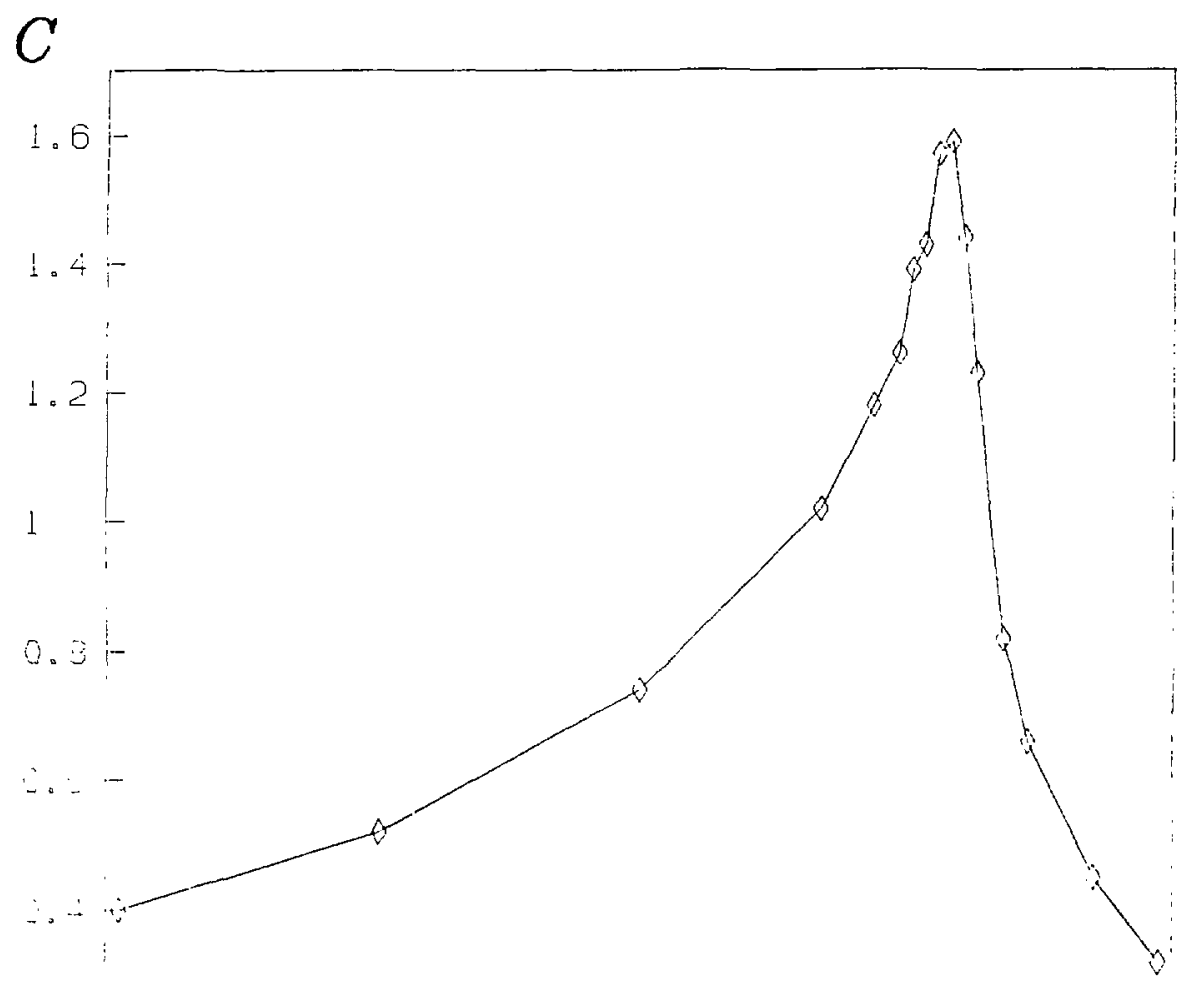

$\therefore 2-$

0

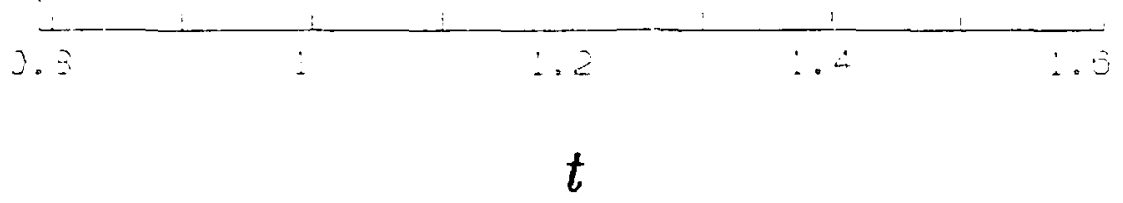

Figure 8 
Thes report was done with support fiom the Department of Energy. Any conelusions or opinions expressed in this report represent salely those of the author(s) and not necessarily those of The Regents of the Liniversity of California, the Lawrence Berkeley Laboratory or the Department of Energy.

Reierence to a company or product name joss not imply approval or recommendation of the preduit by the University of California or ine U.S. Denarment of Energy to the exciusion of others that mạ $b=$ suitab!: 